\title{
Subjectivité de la personne adulte autiste avec retard mental profond, prise en charge dans un établissement d'accueil médico-social
}

\author{
Desnos Yves a \\ GePNER Bruno ${ }^{b}$ \\ Bellusso Perrine ${ }^{c}$ \\ SEGOND Hervé d
}

; Laboratoire de neurobiologie des interactions cellulaires et neurophysiopathologie, UMR CNRS 7259, Université d'Aix-Marseille, France.

c Association Adèle de Glaubitz, Strasbourg, France

d Laboratoire de psychologie des cognitions (LPC) EA4440, Faculté de psychologie, Université de Strasbourg, France.

Correspondance : Hervé Segond, Laboratoire de psychologie des cognitions (LPC EA 4440), Faculté de psychologie, Université de Strasbourg, 12 rue Goethe, 67000 Strasbourg, France.

Courriel
: Nous proposons un cadre théorico-clinique (développemental et systémique), centré sur les théories de l'énaction et de l'intersubjectivité, ainsi qu'une étude individualisée et contextualisée de la cognition des personnes autistes adultes avec déficience intellectuelle profonde, hébergées en structure d'accueil médico-sociale, n'ayant pas accès au langage. La subjectivité de ces personnes est appréhendée en complétant l'évaluation diagnostique par une investigation clinique et expérimentale de leur corporéité, selon certaines modifications préétablies des flux sensoriels et de la relation intersubjective en situation Snoezelen, objectivée à l'aide d'une grille d'observation structurée. L'illustration par un cas clinique souligne l'extrême sensibilité de ces personnes à leur environnement sensoriel et social, ainsi que leur grande vulnérabilité sur le plan de leur intégrité corporelle et psychique. Différents niveaux d'analyse sont proposés en faveur de leur bien-être.

The subjectivity of autistic adults with mental retardation living in institutions

Abstract: We propose a theoretical-clinical framework (developmental and systemic) centered on the theories of enaction and intersubjectivity, and an individualized, contextualized approach to the cognition of autistic adults with profound intellectual disabilities who live in institutions and do not use language. We approach the subjectivity of these people through diagnostic evaluation that investigates their corporeality clinically and experimentally, using pre-established modifications of sensory flows and intersubjective relationships within Snoezelen situations. This is objectified using a structured observation grid. We use a clinical case to emphasize their extreme sensitivity to the sensory and social environment, as well as their vulnerability in terms of bodily and psychic integrity. We present several levels of analysis for their well-being. 


\section{INTRODUCTION}

La loi $\mathrm{n}^{\circ}$ 2005-102 du 11 février 2005, pour l'égalité des droits et des chances, la participation et la citoyenneté des personnes handicapées, est une loi antidiscrimination de portée générale, dont les axes principaux sont de garantir le libre choix du projet de vie, de permettre une participation effective à la vie sociale et de placer la personne au centre du dispositif (Hamonet, 2010). Si les objectifs ainsi définis de l'aide au handicap apparaissent louables et satisfaisants, cette loi reste très générale, vague et englobe, en fait, tout cas dans lequel une personne subirait une incapacité à participer à la vie sociale de son milieu. Elle met ainsi, sur un même plan, tout type de handicap et, par conséquent, normalise les handicaps les plus sévères, notamment ceux pris en charge en Maison d'accueil spécialisée pour personnes handicapées (MAS) ou en Foyer d'accueil médicalisé (FAM), et plus particulièrement les personnes adultes autistes hébergées en structure d'accueil médico-sociale, avec retard mental profond, auxquelles nous consacrons cet article. Il existe très peu de données dans la littérature sur cette population en situation de grande vulnérabilité et de grande dépendance.

Une telle normalisation du handicap s'exerce au détriment du respect qui devrait s'imposer face à une différence manifeste et évidente pour quiconque en fait quotidiennement l'expérience (Chavaroche, 2006). Or, la prise en charge et l'accompagnement de ces personnes adultes avec autisme et retard mental profond associé (AA-DIP) confrontent à des difficultés spécifiques, tant du côté des personnes autistes elles-mêmes, dans leur perception et compréhension de leur environnement physique et social, que de celui de leur environnement social (familial et professionnel), qui a la charge de les aider dans le développement de leur autonomie. Après avoir rappelé la problématique des personnes AA-DIP, nous proposerons une approche théorique et méthodologique de leur subjectivité (point de vue de la personne) en vue de réduire, autant que faire se peut, leurs « situations de handicap » (limitation des activités personnelles ou restriction de la participation à la vie sociale lors de la confrontation du sujet avec la réalité de son environnement physique, social et culturel (voir Hamonet, 2010). Cette proposition, dont nous définirons les principes généraux, est celle de la théorie de l'énaction (initialement proposée par Varela, Thompson, Rosch, 1993), articulant les concepts de corporéité et de cognition incarnée que nous expliciterons plus loin. Cette orientation est issue des résultats des travaux de notre équipe, réalisés, par ailleurs, dans le domaine de la suppléance sensorielle visuo-tactile chez les personnes aveugles (Segond, 2015 ; Segond, Weiss, Sampaio, 2005 ; 2007 ; 2013) et sera illustrée par la présentation d'un cas clinique.

Selon les critères diagnostics actuels de la CIM-11 ${ }^{1}$ (2018), les personnes AA-DIP sont catégorisées comme souffrant de l'interaction entre un trouble du spectre de l'autisme (TSA), dont la catégorie prototypique est celle de l'autisme infantile, et un retard mental profond. Le diagnostic, toujours établi suivant une démarche clinique, repose sur l'existence concomitante des symptômes suivants : troubles de l'interaction sociale et de la communication, comportements restreints et stéréotypés, et précocité des troubles (avant 3 ans), pour ce qui est du syndrome de l'autisme infantile, forte limitation des capacités intellectuelles $(\mathrm{QI}<20)$, de communication et de compréhension, déficiences physiques souvent associées (troubles moteurs, visuels ou auditifs, épilepsie...) et incapacité de subvenir seul à ses besoins fondamentaux, pour ce qui est du retard mental profond.

De ce fait, elles sont sujettes à un multihandicap impliquant les dimensions psychique, mentale et corporelle. Elles sont, à la fois, très vulnérables sur le plan de leur intégrité corporelle et psychique, extrêmement sensibles à leur environnement sensoriel et social, et dramatiquement limitées dans leurs capacités de compréhension d'autrui et des situations, ainsi que dans leurs capacités d'expression, notamment de leurs besoins les plus fondamentaux. Présentant des formes de psychopathologie parmi les plus graves chez l'adulte, ces personnes souffrent toutes de ce que l'on peut définir comme un trouble de l'identité ou une extrême fragilité identitaire, du fait de troubles majeurs entravant l'émergence de l'intersubjectivité et du sens de soi (Bovet, 2005). Ainsi, tandis que toute prise en charge psychologique doit se fonder sur le respect de la subjectivité de ces personnes, conformément au code de déontologie de la profession, cette subjectivité demeure difficilement accessible, occultée par les troubles comportementaux observés et les limitations majeures de compréhension et d'expression, symptomatiques de ce type de pathologie.

1. Classification statistique internationale des maladies et des problèmes de santé connexes, $11^{\mathrm{e}}$ révision, Organisation mondiale de la santé. 
Parallèlement, on ne peut que constater une méconnaissance générale de la problématique de ces personnes en France. Elles font partie d'une population encore très insuffisamment identifiée, recensée, évaluée, accompagnée, prise en charge dans les établissements médico-sociaux et prise en compte dans les politiques publiques, sur les plans quantitatif et qualitatif. Ceci aggrave leur isolement, la violence institutionnelle et la maltraitance dont elles sont parfois l'objet, leurs angoisses et souffrances, leurs comportements inadaptés, leur surmédicalisation et surhospitalisation dans des services psychiatriques inadaptés (Gepner, 2012 ; Recordon-Gaboriaud, 2012). En outre, contrairement aux enfants et adultes autistes de haut niveau, ces adultes autistes très déficitaires sont le plus souvent oubliés des recherches en sciences humaines et sociales, du fait de la difficulté de les évaluer et de les tester.

Ainsi, à l'opposé de la figure du « génie autiste » fortement médiatisée, ces personnes ne semblent offrir a priori aucune compétence spéciale, mais divers « comportements-problèmes », comme le rapporte la Haute autorité de santé (HAS) (2017) dans les recommandations de bonnes pratiques professionnelles (RBP) : comportements archaïques, autostimulations, automutilations, hétéroagressivité, repli sur soi ou manifestations d'angoisse, dont les causes sont, la plupart du temps, très difficiles à identifier. Les comportements observés sont d'autant plus susceptibles d'être négativisés que la parole, plus encore que la marche, signe le statut d'homme (Vaginay, 2007) et que ces personnes sont en majorité non verbales ou n'utilisent la parole que de manière pauvre, stéréotypée, écholalique.

On peut ainsi concevoir toute la complexité et le défi que représente la mise en œuvre d'un accompagnement des sujets AA-DIP en structure médicosociale, qui soit respectueux de leurs différences, attentes, besoins, choix et refus et qui puisse favoriser leur bien-être au quotidien. Ceci implique : $1^{\circ}$ de recourir à une conceptualisation du vécu des sujets correspondant à leur réalité quotidienne, en abordant leur problématique sous un angle positif, en mettant notamment en avant leurs différentes capacités, y compris sociales, plutôt qu'en dressant une liste de leurs incapacités notoires ; $2^{\circ}$ de chercher à accéder à leur subjectivité et à l'objectiver en maîtrisant la nôtre, afin de pouvoir réellement comprendre et respecter leurs différences et leurs choix.

\section{VERS UNE CONCEPTUALISATION COMPRÉHENSIVE DE LA PERSONNE AA-DIP ACCOMPAGNÉE EN STRUCTURE MÉDICO-SOCIALE}

\section{Vers une définition élargie de la problématique autistique}

Depuis la description princeps du syndrome par Kanner (1943), les recherches ont permis d'aboutir à un accord international quant à la nosographie et aux signes cliniques (à l'origine des classifications internationales permettant de poser le diagnostic). Néanmoins, les autismes demeurent encore un mystère face aux questions soulevées par l'étiologie des syndromes, l'identification des mécanismes sous-jacents et l'hétérogénéité des cas cliniques (Frith, 2010 ; Gepner, 2006a ; Hochmann, 1997). Les chercheurs s'accordent aujourd'hui sur le fait que les autismes sont des troubles précoces du développement du système nerveux central d'origine plurifactorielle, multigénique et environnementale. Pourtant, force est de constater l'absence actuelle de consensus, tant au niveau des corpus théoriques sous-tendant les divers champs de recherche, qu'au niveau des programmes éducatifs et des approches thérapeutiques (Chamak, Cohen, 2003 ; Pelloux, Delion, Tessarech, 2010 ; Tardif, Gepner, 2014).

Depuis quelques années, la publication et la diffusion des témoignages de personnes Asperger et autistes de haut niveau ont ouvert le champ à des descriptions du syndrome selon un point de vue « à la première personne ». Comme le suggèrent Chamak, Bonniau, Jaunay et Cohen (2008), ces descriptions de l'autisme « depuis l'intérieur » nous invitent à une vision élargie et à une redéfinition de l'autisme où les troubles de la dyade autistique (troubles de la communication sociale, comportements restreints et répétitifs - CIM-11, 2018 ; DSM-5 ${ }^{2}$, 2013) sont les conséquences de troubles neuropsychologiques plus primaires : altération des systèmes sensoriels, atypicité des processus de traitement de l'information (saillance des détails plutôt que de la forme globale) et troubles de la régulation émotionnelle (Chamak et coll., 2008). Ces trois dimensions explicatives de la symptomatologie autistique sont à présent fortement étayées par la littérature scientifique, tant sur le plan clinique qu'expérimental (voir Cascio, 2010, Iarocci, MacDonald, 2006 et Tardif, 2010, pour l'altération des systèmes sensoriels ; Nadel, 2003 et Thommen, 2010 pour les troubles

2. Diagnosis and Statistical Manual of Mental Disorders, $5^{\mathrm{e}}$ édition révisée, APA (American Psychiatric Association), 2013. 
de la régulation émotionnelle ; Happé, Frith, 2006 et Mottron, Dawson, Soulières, Hubert, Burack, 2006, pour l'atypicité des processus de traitement de l'information).

Selon l'hypothèse explicative générale, proposée par Gepner (2006a, 2006b), ces troubles cognitifs primaires peuvent eux-mêmes être considérés comme résultant d'un désordre initial du traitement temporospatial des flux et événements sensoriels. Ces troubles d'intégration temporo-spatiale des informations multisensorielles résulteraient d'un phénomène de « dyssynchronie cérébrale multisystème » (au sein ou entre des réseaux neuronaux et des voies neurofonctionnelles plus ou moins distants) : cette dyssynchronie (définie comme un défaut ou un excès de synchronisation électrique entre des assemblées de neurones) affecterait initialement un ou plusieurs « systèmes-clefs » (notamment les systèmes visuel ou auditif magnocellulaires, le cervelet, la voie visuelle dorsale, le sillon temporal supérieur et les neurones miroirs) avant de s'étendre éventuellement plus largement par la suite. Une « dysconnectivité cérébrale multi-système » (hypo ou hyperconnectivité) au sein d'un réseau neuronal ou entre des réseaux fonctionnels multiples serait la contrepartie spatiale de ce phénomène. L'hypothèse selon laquelle cette dysconnectivité cérébrale, aux manifestations variables selon les sujets, puisse être considérée comme l'un des troubles neuro-bio-physio-psychologiques de base des troubles autistiques, apparaît fortement étayée par de nombreuses recherches actuelles en histologie, électrophysiologie et neuro-imagerie (voir, pour revue, Wass, 2011). Ainsi, selon Gepner (2006a, 2006b), ces phénomènes précoces de dyssynchronie et dysconnectivité cérébrale multi-systèmes, occasionnant des troubles d'intégration temporo-spatiale des flux ou événements sensoriels, aux premiers stades du développement, pourraient expliquer, par le biais de « cascades mal-développementales », un nombre important de déficits et de particularités rapportés comme significatifs dans le syndrome : évitement sensoriel (quand le flux sensoriel est aversif), évitement social, découplage perceptionaction, désaccordage sensorimoteur, désordres de compréhension verbale et émotionnelle, anomalies de compréhension du monde physique et humain, désaccordage social et affectif (Gepner, 2006a).

De manière générale, ce modèle théorico-clinique permet de comprendre les syndromes de l'autisme comme des troubles précoces majeurs du fonctionnement et du vécu corporel en situation, entraînant, d'une part, des altérations « en cascade » des fonctions clefs (sensorielle, motrice, émotionnelle, communicationnelle, sociale, psychique, etc.) et, d'autre part, l'émergence de stratégies compensatoires (à l'origine des nombreuses particularités de fonctionnement). Ce modèle permet également de comprendre les différences de vécu et d'expérience subjective que ces personnes éprouvent face à leur environnement. En outre, il apparaît utile sur le plan thérapeutique, puisque des résultats d'études expérimentales et translationnelles ont montré, de manière consistante, les bénéfices du ralentissement des signaux audio-visuels sur l'attention visuelle, la cognition verbale, le comportement (comme la communication et la réciprocité sociale) et le développement d'enfants autistes (Gepner, 2014 ; Tardif, Latzko, Arciszewski, Gepner, 2017).

Par conséquent, la problématique des personnes AA-DIP peut être caractérisée par : $1^{\circ}$ une différence qualitative du développement, liée à un développement cérébral, corporel, mental et psychique atypique depuis le début de la vie $; 2^{\circ}$ un trouble de l'intégrité corporelle ayant entraîné des stratégies d'adaptation/de compensation ; $3^{\circ}$ une incapacité à s'exprimer sur leur vécu. Se pose alors une question essentielle, à la base de toute prise en charge de ces sujets : comment donner sens à leurs comportements en contexte ? Autrement dit, comment appréhender leur subjectivité ?

Pour ce faire, nous nous sommes référés à certains concepts issus de la théorie de l'énaction, ainsi qu'au champ théorique de l'intersubjectivité, la complémentarité de ces perspectives nous paraissant en adéquation avec ces questionnements.

\section{Apport du concept d'énaction}

La théorie de l'énaction, initialement développée par Varela et coll. (1993), propose une compréhension de la cognition humaine suivant une perspective écologique, qui souligne la nature biologique des êtres humains (Hutchins, 2010). Tout d'abord, cette théorie réfute l'idée d'un monde extérieur au sujet, aux propriétés prédéfinies : le sujet et son environnement se co-constituent et s'inter-spécifient, au gré de l'évolution considérée comme dérive naturelle (Varela et coll., 1993). Sebbah (2004) précise que le paradigme de l'énaction donne ainsi accès à une « voie moyenne », selon laquelle ce qui est « premier»n'est ni le sujet, ni l'objet, mais la relation dynamique dans laquelle ils se spécifient mutuellement.

La théorie de l'énaction propose de considérer que l'action du corps en contexte de tout être 
vivant est à l'origine de sa subjectivité et de son expérience : la cognition est dite « incarnée », elle est toujours «située » dans l'interaction entre le corps et le monde. Autrement dit, la conscience ou subjectivité d'un individu relève, en premier lieu, de son vécu corporel en situation. La pensée peut être considérée comme une émergence de l'expérience corporelle.

Les travaux que certains d'entre nous ont menés auprès de personnes aveugles sur la suppléance sensorielle tactile d'informations visuelles, sur la plasticité corticale et cognitive, nous ont conduits à considérer ce cadre théorique comme particulièrement pertinent pour constater combien les processus corporels dynamiques (comme l'activité sensori-motrice) prennent part aux processus de raisonnement (Segond, 2015 ; Segond et coll. 2005, 2013). Varela et coll. (1993) ont proposé la notion de corporéité (embodiment), pour désigner le corps, à la fois, comme structure vécue et comme contexte ou lieu des mécanismes cognitifs. Ce concept souligne l'indissociabilité de l'expérience de l'individu, de son vécu corporel en situation et de sa structuration cognitive, laquelle émerge au gré de l'histoire de ses interactions avec son écosystème. Le cerveau possède la capacité de s'adapter au changement en s'organisant structuralement, au cours des interactions et expériences de l'organisme au sein de son environnement physique et social, dans le cadre du développement normal ou bien en se réorganisant dans le cadre de situations pathologiques ou accidentelles (lésion cérébrale, privation sensorielle). La mise au point de procédures d'apprentissage intensif spécifiques a permis à des personnes aveugles de percevoir des objets tridimensionnels stables à distance, comme étant situés devant eux, reflétant ainsi le phénomène de mise en extériorité des percepts (caractéristique de la suppléance sensorielle visuo-tactile), mais, également, des informations spécifiquement visuelles, comme la profondeur, la perspective ou la parallaxe de mouvement (Segond, 2015 ; Segond et coll., 2005, 2013). Le phénomène de mise en extériorité était renforcé par le sentiment d'implication subjective dans l'espace exploré (par exemple, un labyrinthe, exploré à l'aide d'un robot radiocommandé par les sujets, en fonction des indices tactiles transmis par l'intermédiaire de la caméra embarquée du robot). La nature de la spatialisation chez ces sujets (localisation subjective d'objet et de soi-même dans l'espace), qui se manifeste à travers ce sentiment d'explorer un espace dans lequel ils se perçoivent impliqués, tout en n'étant pas physi- quement là où se meut le récepteur, qui fournit les informations de type visuel, se conçoit à partir de la théorie de l'énaction. L'émergence de la structure cognitive de l'individu s'opère progressivement au cours de son développement, à partir de l'utilisation répétée de ses schèmes sensorimoteurs en situation. C'est ainsi l'expérience/la structure sensorimotrice incarnée en contexte (la corporéité) d'un individu que l'on peut considérer comme étant à la base de sa subjectivité, tant sur le plan de son vécu que de son développement cognitif singulier.

\section{Primat de la dimension sociale}

\section{et de l'intersubjectivité dans la corporéité}

Chez l'être humain, l'ontogénèse de la sensorimotricité, constitutive au premier plan de la corporéité de tout être vivant, apparaît tributaire et indissociable de la dimension sociale. Une partie conséquente de la recherche théorique et expérimentale en psychologie du développement et en sciences cognitives amène ainsi à comprendre que les fondements de la cognition humaine impliquent des processus interactionnels de nature sociobiologique.

Dans ce cadre, le courant dit de la « cognition incarnée » conduit à considérer l'esprit, non plus comme un ensemble de fonctions logiques et abstraites, mais comme un système biologique enraciné dans l'expérience corporelle et interconnecté avec l'action corporelle et l'interaction avec les autres individus (Garbarini, Adenzato, 2004). Plus précisément, l'individu humain, en présence d'autrui, apparaît spontanément connecté aux comportements de l'autre : l'observation du comportement d'autrui active automatiquement son système moteur, comme s'il faisait lui-même directement l'expérience au niveau corporel de ce qu'il observe, qu'il s'agisse d'actions envers les objets, les personnes ou, surtout, d'expressions émotionnelles (Garbarini, Adenzato, 2004 ; Niedenthal, 2007; Rizzolatti, Fabbri-Destro, Cattaneo, 2009 ; Rizzolati, Sinigaglia, 2008 ; Winkielman, McIntosh, Oberman, 2009). De ce fait, des individus en présence sont inévitablement sensibles à un phénomène de contagion motrice et émotionnelle (Grèzes, De Gelder, 2005).

La cognition sociale de l'individu humain apparaît reposer, en première instance, sur un phénomène dynamique, que Fuchs et De Jaeger (2009) nomment « intersubjectivité énactive » : ce phénomène consiste en une « incorporation mutuelle » entre les individus en présence, processus par lequel les corps de chacun (compris dans leur dimension existentielle vécue par les individus, leurs corporéités) se rejoignent et 
forment une « intercorporéité commune ». L'intersubjectivité est ainsi conçue comme un processus « d'interaction incarnée », qui préexiste à la volonté consciente des individus et génère une signification commune et partagée entre eux.

Ces approches prolongent et complètent de nombreux travaux en psychologie du développement soulignant l'importance fondamentale du processus d'intersubjectivité dans le développement humain. Nous citerons, notamment, ceux du groupe de recherche de Trevarthen (voir Aitken, Trevarthen, 2003 ; Trevarthen, 2003 ; Trevarthen, Aitken, 2003), qui postule l'existence, chez les enfants autistes, d'un trouble développemental de l'intentionnalité et de l'émotionnalité en situation sociale, du fait d'un dysfonctionnement de la « formation motivationnelle innée » ou «formation intrinsèque de motivation » (Intrinsic Motive Formation, IMF), composée de plusieurs noyaux du tronc cérébral. L'IMF régule la coordination des images motrices et de l'activité efférente des membres et du corps tout entier, ainsi que les ajustements moteurs et les expériences ; en conversation proche, l'activité motivationnelle et émotionnelle dynamique d'un sujet s'exprime principalement par des mouvements du visage, des yeux, du système vocal et des mains (impliquant notamment les modalités visuelle, auditive et tactile), dont la synchronisation, tant sur le plan expressif que réceptif, dépend du fonctionnement de l'IMF. La synchronisation dynamique précoce (au stade de l'intersubjectivité primaire) des systèmes IMF d'un nourrisson et de sa mère (ou autre pourvoyeur de soins), est nommée protoconversation. Trevarthen et Aitken (2003) postulent l'existence d'un « état initial psychosocial » chez le nourrisson, l'entraînant à communiquer, dès le premier semestre de vie, par protoconversation avec les formes expressives et rythmiques d'intérêt et d'émotion manifestées par autrui. Les émotions présentent ainsi une double fonction : permettre la régulation biologique du nourrisson, en signalant ses besoins corporels et en provoquant une réponse parentale adaptée, et fournir un support à la communication préverbale (protoconversation) et à l'apprentissage culturel, induisant l'émergence de la conscience de soi. L'ensemble du développement de l'enfant (dans les dimensions biologique, sociologique et psychologique) repose ainsi initialement sur l'expressivité émotionnelle de ses intérêts et intentions, et la perception de celle de ses partenaires, selon une dynamique interactionnelle et développementale.
Ce processus d'attention mutuelle, nommé « intersubjectivité primaire », induit et régule les comportements de synchronisation affective et motrice entre le bébé et l'adulte (accordage affectif). Il permet, notamment, l'émergence, dès le second semestre de la première année, de l'intersubjectivité secondaire, où les conduites, jusque-là centrées sur le face à face, intègrent un objet extérieur sur lequel porte l'attention conjointe, un objet commun auquel les partenaires font, l'un et l'autre, référence, impliquant le recours à des conventions relatives aux moyens d'expression utilisés. Le groupe de recherche de Cosnier (voir Cosnier, 1981; Cosnier, Chavarel, 1998) souligne également le primat de l'intersubjectivité et de la communication intercorporelle dans l'ontogénèse de l'individu humain, à travers la notion d' "épigénèse interactionnelle », conçue en tant qu' « auto-organisation éco-dépendante » (Cosnier, Chavarel, 1998).

Suivant ces approches, l'enfant et ses figures d'attachement s'interspécifient continuellement, à travers un processus d'intersubjectivité primaire, tout d'abord médiatisé par le corps de chacun et la communication émotionnelle et affective, bien avant l'émergence de la parole et de la pensée. De plus, comme le mettent en évidence les recherches dans le champ de la cognition incarnée, ce processus d'intersubjectivité primaire constitue également la base de la cognition de l'individu adulte en contexte social.

Ainsi, Kaufmann et Clément (2003), à partir d'une revue de nombreux arguments issus de l'éthologie, de la psychologie évolutionniste et de la psychologie du développement, soutiennent qu'il est aujourd'hui nécessaire de redéfinir la constitution de l'architecture cognitive de l'être humain, en considérant que l'ordre des faits sociaux (les interactions ou relations sociales) précède, chez l'homme, l'ordre des faits psychologiques (les capacités représentationnelles ou mentales des individus), tant sur le plan de la phylogénèse de l'espèce que de l'ontogénèse de l'individu.

\section{La corporéité des sujets comme base de compréhension de leur subjectivité}

Du fait de la différence qualitative de vécu et de fonctionnement sous-tendue par la pathologie des sujets, il semble judicieux, tant sur le plan éthique qu'épistémologique, d'aborder leur subjectivité via la notion de corporéité. Tenter de comprendre leur subjectivité à partir d'un plan phénoménologique, fondé sur l'observation des comportements en situation, permet de recueillir des informations observables et discutables, tout en limitant nos 
propres projections et présupposés sur la nature de leur cognition. Parallèlement, ce choix théorique et méthodologique nécessite un exercice d'auto-analyse sur la nature de notre propre corporéité, ainsi qu'une observation et une réflexion critiques de notre propre fonctionnement en contexte.

Suivant la théorie de l'énaction, les comportements des sujets participent, en premier lieu, à leur autopoïèse (Chamak, 2004), un état d'équilibre ou d'homéostasie par l'action, au cours des relations entretenues entre tout être vivant et les autres systèmes complexes de son environnement (son écosystème). Leur sens et intentionnalité résultent de l'histoire de leurs interactions avec leur environnement. Ainsi, aborder la subjectivité des sujets via leur corporéité implique l'investigation de leur structure sensorimotrice, c'est-à-dire la manière dont les comportements des sujets contribuent à maintenir leur organisme dans un état d'équilibre, selon leur histoire développementale et les modifications du contexte. Dans cette perspective, nous nous référons aux travaux de Thommen et Chastellain (2009), pour qui les stéréotypies sensorimotrices des sujets adultes autistes (fréquentes dans cette population, car liée au degré d'autisme et de retard mental), ont pour fonction principale l'autorégulation corporelle, mais de manière différenciée selon les individus et les situations : une même stéréotypie sensori-motrice (comme un comportement de balancement) peut avoir une fonction d'autostimulation pour certains ou de protection/ pare-excitation ${ }^{3}$ pour d'autres (Bourreau, 2008 ; Thommen, Chastellain, 2009) et peut résulter de modifications sensorielles ou sociales de leur environnement.

De manière générale, les stéréotypies des sujets nous sont apparues comme des marqueurs fondamentaux de la corporéité des sujets, en ce sens qu'elles pouvaient manifester leurs vécus corporels et émotionnels (intensité, fréquence), ainsi que leur investissement respectif des différentes dimensions sensorielles.

\section{Primat de la relation sujet-accompagnant pour appréhender la cognition des sujets}

Outre l'importance de la prise en compte de la dimension sensorielle et sensorimotrice dans

3. Dans la théorie freudienne, le système pare-excitation traite et gère les excitations, et vise à maintenir l'équilibre interne, l'homéostasie du sujet. Ce terme apparaît chez Freud dans Au-delà du principe de plaisir, en 1920 (Laplanche, Pontalis, 1967). l'étude de la corporéité des sujets, nous soulignons également la nécessité d'aborder leur cognition via la dimension sociale et relationnelle, en mettant au premier plan leurs modalités d'expressivité émotionnelle. En effet, les troubles de l'interaction sociale et de la communication n'impliquent pas une absence de volonté de communiquer et encore moins une insensibilité aux interactions sociales. À l'inverse, comme l'ont montré Nadel et Potier (2002) dans leurs travaux sur la sensibilité des sujets AA-DIP à l'imitation de leurs stéréotypies sensorimotrices, ces personnes cherchent activement le contact avec autrui, à partir du moment où cela leur est possible.

Selon la théorie de l'énaction, les sujets sont indissociables de leur contexte au sein duquel ils se développent, en fonction des relations de spécification mutuelle existantes avec les individus et autres systèmes composant leur environnement. Dans ce cadre, il nous paraît fondamental de prendre en compte, en premier lieu, la relation sujet-accompagnants pour appréhender la cognition des sujets. En effet, du fait de leur situation de grande dépendance, le bien-être des sujets est directement tributaire des comportements des accompagnants à leur égard.

\section{ILLUSTRATION CLINIQUE}

\section{Cadre de l'étude}

Cette recherche clinique a été conduite pendant quatre ans au sein d'un foyer d'accueil médicalisé (FAM) en Alsace (67). Officiellement reconnus depuis la loi $\mathrm{n}^{\circ}$ 2002-2 du 2 janvier 2002, rénovant l'action sociale et médico-sociale, les FAM sont destinés à accueillir des personnes adultes lourdement handicapées, dont la dépendance totale ou partielle, constatée par la Commission des droits et de l'autonomie des personnes handicapées $(\mathrm{CDAPH})$, les rend inaptes à toute activité à caractère professionnel, leur fait obligation de recourir à l'aide d'un tiers pour la plupart des actes essentiels de la vie quotidienne et nécessite parfois une surveillance médicale et des soins constants. Ces structures ont vocation à prendre en charge, à temps complet, les personnes accueillies (« usagers » ou « résidents ») et peuvent également pratiquer l'accueil de jour permanent ou l'accueil temporaire, pour un nombre limité de places (DREES, 2010).

Elles diffèrent des structures hospitalières et psychiatriques, dans la mesure où elles constituent un lieu de vie quotidien et à long terme pour la population accueillie. La moitié de celle-ci est constituée d'adultes polyhandicapés et l'autre 
moitié d'adultes avec une déficience intellectuelle profonde ou sévère, laquelle est associée, selon les cas, à un trouble envahissant du développement (TED), à un trouble psychotique, ou au syndrome de Down (trisomie 21), ainsi que, parfois, à des troubles somatiques (épilepsie) ou sensoriels (cécité, surdité).

L'organisation de la structure repose, en grande partie, sur le fonctionnement au quotidien des groupes de vie. Chaque groupe consiste, en moyenne, en l'accompagnement de dix sujets par huit accompagnants, intervenant par équipe de deux ou trois par demi-journée (suivant un système de rotation). Le vécu des résidents est ainsi quasiconstamment relatif à des situations de groupe et les situations sociales sont souvent très complexes, puisqu'elles impliquent, sur une base de douze personnes en présence, des modifications et perturbations fréquentes (départs et entrées sur le groupe, absences, etc.).

La recherche menée au sein du FAM impliquait d'être au croisement des champs clinique et scientifique, de conserver une neutralité de principe par rapport aux relations hiérarchiques établies (par exemple, les relations cadres-accompagnants), tout en respectant l'éthique institutionnelle et en s'adaptant continuellement au fonctionnement de la structure. En conséquence, le fondement de notre démarche a consisté en une immersion au sein du FAM (méthodologie de l'observation participante), en ayant, durant nos temps de présence, une attitude clinique non directive à l'égard des sujets (investigations psychologique et clinique selon leurs problématique et leurs interactions), tout en interagissant avec l'ensemble des personnes en présence. Cette attitude nous a amenés à développer ce que l'on pourrait décrire comme une ethnométhodologie de l'autisme dans ce type de structure.

Ainsi, notre démarche peut être décrite en tant que recherche clinique, de type recherche-action (voir Calpini et coll., 1981), où l'intention du clinicien-chercheur est posée au départ - objectiver la subjectivité/réduire la situation de handicap des sujets concernés -, mais où la modélisation théorique, la méthodologie et le cadre de la recherche se construisent en partie au fur et à mesure des résultats observés. Cette démarche, qui peut avoir un caractère aventureux voire indéterminé, nous a paru cependant appropriée pour aborder, de manière éthique et épistémologiquement valide, la problématique de ces sujets atteints de troubles extrêmement sévères et complexes, très démunis sur le plan fonctionnel et communicationnel et très sensibles à leur environnement, lui-même mouvant et complexe.

Ce faisant, notre démarche s'apparente à celle de la recherche systémique en psychologie clinique (Courtois, 2009), caractérisée par : $1^{\circ}$ un abord contextualisé de l'être humain et de sa réalité complexe (individu, organisations, milieux, relations) ; $2^{\circ}$ une démarche complémentaire de subjectivation (centrée sur le sujet au cour de l'intervention) et d'objectivation (du vécu/ressenti des sujets et des effets de nos interventions) de l'objet d'étude ; $3^{\circ}$ une attitude propositionnelle et non-directive de la part du chercheur ; $4^{\circ}$ une co-construction de l'objet d'étude avec les personnes qui collaborent à la recherche ; $5^{\circ} 1^{\prime}$ 'émergence de modèles mis à l'épreuve des situations ; $6^{\circ}$ une ouverture conceptuelle et le développement d'une multi-méthodologie (recours à des indicateurs multiples permettant de trianguler les sources d'informations). En fait, on peut considérer que cette étude, conduite suivant une démarche écologique, combinant démarche clinique et expérimentale en psychologie, constitue une opérationnalisation exploratoire du paradigme de l'énaction en psychologie du développement, accordant une importance toute particulière aux notions de corporéité et d'interspécification cognitive, d'intercorporéité et d'énaction intersubjective, concepts qui nous semblent à même de retranscrire de manière adaptée les dimensions fondamentales relatives à la problématique des sujets.

Nous présentons, dans cet article, les principes généraux de la méthodologie employée pour dresser un profil corporel de chaque sujet pris en charge dans le cadre de cette recherche appliquée. Nous illustrerons cette méthodologie par la présentation d'une étude de cas.

\section{Formalisation du profil corporel individuel}

Cette étape de la recherche a consisté en la formalisation de ce que nous avons nommé un profil corporel (suivant la méthodologie de l'étude de cas unique ; voir Smith, 2012). Celui-ci a pour objectif de dresser un profil sensoriel, émotionnel, communicationnel et relationnel de chaque sujet.

La méthodologie, que nous avons élaborée à cette fin et proposée à cinq sujets AA-DIP, répartis au sein de trois des groupes de vie, a consisté en une investigation de leur corporéité, à partir de la triangulation d'informations opérée entre : $1^{\circ}$ les informations obtenues par l'analyse de prises en charge individuelles non directives semi-standar- 
disées, analysées a posteriori par analyses vidéo. Celles-ci avaient notamment pour but d'objectiver, dans le cadre d'une approche Snoezelen, présentée plus loin, les comportements intersubjectifs intentionnels, les fonctions des stéréotypies sensorimotrices observées et la réactivité des sujets selon le contexte sensoriel ; $2^{\circ}$ celles relatives à l'évaluation diagnostique des sujets, à leur anamnèse et à l'évaluation de leur degré d'autisme (CARS-T, Childhood Autism Rating Scale, échelle d'évaluation de l'autisme infantile ; Schopler et coll., 1980/1989) et leur niveau de développement socio-adaptatif dans les domaines de la communication, de l'autonomie, de la socialisation et de la motricité par les échelles Vineland (VABS, Vineland Adaptative Behavior Scales ; Fombonne, Achard, Tuffreau, 1984/1995). L'évaluation des sujets via la CARS et les VABS a été réalisée auprès de leur accompagnant référent respectif, au cours d'un entretien semi-directif spécifique.

Nous avons ainsi cherché à mettre en évidence les spécificités respectives des sujets AA-DIP sur : $1^{\circ}$ leurs modalités d'expressions émotionnelles (valence positive et négative) ; $2^{\circ}$ leurs modalités de communication intentionnelle intercorporelle ; $3^{\circ}$ leurs recherches actives de sensations (stratégies d'autostimulation ou de parexcitation) ; $4^{\circ}$ leurs réactivités sensorielles atypiques (hyposensibilité/

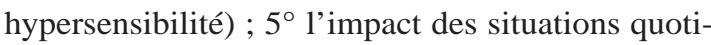
diennes (valence négative ou positive).

Nous avons, en parallèle, effectué une enquête auprès du personnel accompagnant (questionnaires d'auto-évaluation de la prise en charge du résident). Nous avons ainsi élaboré un questionnaire en deux parties : la première ayant pour objectif de dépeindre la dimension socio-affective du cadre d'accompagnement d'un sujet, la seconde de recueillir des informations plus spécifiques sur sa corporéité au quotidien.

Nous avons également choisi d'étudier, au niveau des équipes, certaines représentations sociales pouvant nous permettre d'affiner notre compréhension du contexte dans lequel évoluent les sujets AA-DIP. En effet, comme le rapporte Abric (2003), une représentation sociale est une appropriation, une reconstruction par les individus et les groupes d'une réalité, intégrée dans leur système de valeur, dépendant de leur histoire et du contexte social et idéologique qui les environne ; une représentation sociale constitue ainsi un système sociocognitif guidant l'action, orientant les relations, les communications et les pratiques sociales. Ainsi, dans les milieux professionnels, les représentations sociales et les pratiques professionnelles s'engendrent mutuellement et ne peuvent être dissociées.

Ces données d'enquête visent, à terme, à proposer une intervention systémique psycho-éducative aux équipes, leur permettant d'améliorer leur fonctionnement au quotidien, certains accompagnants se disant en souffrance de ne pouvoir réaliser de manière appropriée un accompagnement éthique et adapté à chacun. Pour illustrer ces difficultés, nous avons été saisis par le contraste entre l'attitude de repli adoptée la majorité du temps par Sylvie ${ }^{4}$ (la résidente dont nous présentons le profil corporel plus loin) au sein de son groupe de vie (assise, statique, ne recherchant pas le contact) et son dynamisme, son plaisir à communiquer, lors de nos séances de prise en charge. Les réponses des accompagnants aux questionnaires ont plusieurs fois mis en évidence une forte disparité des modalités d'intervention auprès d'elle (fréquence, temporalité, méthode), ce que nous a confirmé notre analyse clinique en situation. De plus, nous avons observé, lors des situations d'entretien, une grande variabilité chez ces derniers quant à leur connaissance ou méconnaissance de son fonctionnement et vécu corporel au quotidien (une majorité des accompagnants avait notamment des difficultés à expliciter leurs réponses et à donner des exemples).

La méthodologie et les résultats de ces tests de représentation sociale du personnel accompagnant (relatives aux notions d'autisme, de prise en charge et de sensorialité) et de l'intervention systémique seront présentés dans d'autres articles ultérieurement.

Soutenant une perpective systémique et énactive du handicap, généré par le syndrome, nous considérons que la triangulation et la complémentarité des points de vue et des techniques d'investigation sont nécessaires et peuvent permettre d'atteindre une certaine objectivation consensuelle du fonctionnement des sujets.

\section{Approche Snoezelen}

Notre approche et notre attitude envers chaque résident a été définie, dès l'origine, comme participant à l'approche Snoezelen. Initiée par Hulsegge et Verheul (1988), celle-ci se démarqua radica-

4. Conformément à l'article 26 du code de déontologie des psychologues (version actualisée de février 2012 accessible à l'adresse : http://www.codededeontologiedespsychologues.fr/LE-CODE.html) le prénom du sujet a été changé dans le respect absolu de l'anonymat. 
lement des activités psychothérapeutiques traditionnelles de cette époque, par la création d'un environnement non angoissant et sécurisant, dans lequel des enfants ou des adultes déficients, ou avec d'autres limitations, peuvent profiter agréablement de situations sensorielles adaptées. Le mot « snoezelen » est une contraction des verbes hollandais snuffelen (rechercher et explorer) et doezelen (se relaxer). Au-delà de la création de salles équipées ou d'espaces adaptés, cette approche peut être définie comme ouverte, sensible, attentionnée, non directive, dans laquelle une atmosphère de sécurité est créée et le libre choix encouragé. Les notions de non-directivité et d'expérience partagée apparaissent centrales dans la philosophie originelle : les personnes concernées sont, pour un temps, libérées des attentes de leur contexte, ainsi que de la pression liée aux soins préétablis. La volonté des auteurs était de ne pas donner à la thérapie et au développement une place centrale, tout en laissant ouvertes les possibilités d'utilisation.

Malgré l'absence de référentiels théoriques spécifiques, la démarche Snoezelen apparaît actuellement en plein essor, comme le montrent les nombreux colloques organisés sur ce sujet dans différents pays (Belgique, Suisse, Allemagne, Canada, France, etc.), ainsi que par la multiplication de ses champs d'application : enfants et adultes polyhandicapés, enfants et adultes autistes et psychotiques, personnes âgées démentes, personnes en fin de vie, malades atteints du VIH, personnes en phase d'éveil de coma (Blondel, 2003).

L'approche Snoezelen peut être utilisée dans une optique de relaxation ou de stimulation ; elle peut consister en des expériences multisensorielles ou focalisées sur une seule stimulation, par la simple adaptation de l'environnement (lumières, sons, odeurs, textures...) aux besoins spécifiques de la personne, le temps de la prise en charge. Ainsi, s'agissant plus spécifiquement de la prise en charge des sujets autistes, l'utilisation de cette démarche peut permettre d'améliorer leur état général, grâce à l'apaisement que l'adaptation de l'environnement à chaque problématique peut permettre ; sur la base de cet apaisement et du sentiment de sécurité suscité, elle peut, de plus, offrir un espace permettant d'apprendre à apprendre, de favoriser les explorations sensorielles, de développer les communications interpersonnelles grâce à un meilleur éveil à l'autre et à soi-même, ou d'offrir à la personne un espace de récupération après une trop lourde charge sensorielle, cognitive, sociale ou émotionnelle (Tardif, 2010).
Les principes constitutifs de cette démarche apparaissent, en premier lieu, d'ordre éthique et philosophique, car ils réinterrogent la relation entre professionnel du secteur médico-social et sujet handicapé. Comme le souligne Blondel (2003), le Snoezelen consiste à proposer un temps « d'activité » dans un environnement qui propose des sollicitations sensorielles ayant en commun d'amener des sollicitations sensorielles douces, diversifiées, où tous les sens pourront être sollicités séparément ou simultanément : vue, toucher, odorat, sens de l'équilibre (vestibulaire), audition. Le climat relationnel est un élément à part entière du Snoezelen. L'objectif de cette relation est de limiter le stress, de permettre, de favoriser l'expression d'un choix de personnes, dont la motricité et la capacité à communiquer et à se faire comprendre sont limitées et pour lesquelles la mise en sens revêt des formes très singulières (parfois, cette liberté de choix ne peut s'exprimer que par le refus ou l'éviction de la relation), de respecter le rythme de la personne accompagnée (laisser le temps à ces personnes de répondre ou d'agir à leur manière, de leur accorder du temps pour tenter de comprendre leurs modalités d'expression si particulières). Au travers de cette définition, le Snoezelen apparaît, à la fois, comme un lieu, une activité, une philosophie : une façon de penser l'autre différent comme sujet, une activité qui participe à la «production de la dignité » et à la « mise en humanité » de la personne.

L'approche Snoezelen se prête ainsi remarquablement à notre propre conception éthique des sujets AA-DIP, tout comme elle fournit un cadre propice à l'opérationnalisation clinique des concepts issus de la théorie de l'énaction développés précédemment.

Ainsi, nos prises en charge Snoezelen ont, premièrement, consisté à agir sur le rapport de présence entre les sujets AA-DIP et nous-même, d'une manière propositionnelle et adaptative, sur la base de nos observations en temps réel des actions corporelles des sujets nous amenant à inférer leur vécu en situation (valence positive ou négative). Nous nous sommes ainsi adaptés aux actions des sujets, selon que nous les interprétions comme des comportements d'approche (comme prendre la main), d'évitement (pousser des cris stridents, par exemple) ou des comportements ambivalents, ceci étant le cas de la majorité des stéréotypies corporelles, en raison de la double fonction possible des stéréotypies mentionnées précédemment.

Les principes de liberté de choix (attitude non directive et propositionnelle de notre part) et de 
respect de la temporalité de la personne accompagnée et de ses rythmes ont impliqué que nous adaptions notre comportement à celui du sujet pris en charge, afin que celui-ci puisse se considérer comme acteur à part entière de la relation, ses choix éventuels étant reconnus. Ce faisant, nous avons adopté une position symétrique avec les sujets, dès que cela était possible (même statut, compagnonnage), bien qu'un positionnement complémentaire soit parfois apparu nécessaire (protection, comme dans le cas de crises d'anxiété, éventuellement autorité, comme dans le cas de comportements sexuels inappropriés).

Nous avons conçu chaque prise en charge comme une rencontre, une situation d'intercorporéité, reposant, notamment, sur le principe de synchronisation intercorporelle (impliquant les plan affectif et sensorimoteur) ; nos actions envers les sujets ont ainsi consisté, d'une manière individualisée pour chaque personne, à chercher à susciter le bienêtre des sujets, tout en étayant les mécanismes de synchronisation intercorporelle, sur la base de nos observations en temps réel des actions des sujets, ceci nous permettant d'inférer prospectivement comment chaque personne utilisait de manière spécifique ses modalités sensorielles en situation.

Nous avons ainsi principalement porté attention à notre utilisation du déplacement (régulation de la distance), du positionnement du buste (faire face, approcher sur le côté, tourner le dos), du regard (croisement visuel, regard périphérique, évitement), de la voix (sons, chant, parole, silence), et bien sûr du toucher (pression, vibration, toucher fin). Lorsque nous interprétions que les sujets abordaient la relation proposée suivant une valence affective positive (ou, au moins, non négative), nous avons cherché à leur proposer d'entrer davantage en contact et de développer une réciprocité des échanges. Nous avons, dans ce cadre, utilisé l'imitation de certaines stéréotypies sensori-motrices (imitation de sons stéréotypés, par exemple) ou initié, de manière respectueuse, certains contacts directement via notre corps (rapprochement, regard, voix, toucher) ou par la médiation de différents objets ayant diverses propriétés sensorielles, les sujets ayant toujours le choix d'investir ou non la relation. Enfin, la dimension temporelle a constitué un axe essentiel de notre approche des sujets AA-DIP : respect de leurs rythmes personnels, souci des transitions (comme sortir du lieu de vie), synchronisation de notre comportement au leur ; ceci nous apparaissant fondamental en référence aux hypothèses de la problématique autistique rappelées précédemment (troubles d'intégration temporo-spatiale des flux ou événements sensoriels et dysfonctionnement de l'IMF). Nous rapprochons notre approche personnelle du Snoezelen de la notion de zone proximale de développement, initialement développée par Vygotski (1985) : en effet, «l'espace relationnel», suscité suivant notre démarche (que l'on pourrait globalement qualifier de méta-synchronisation corporelle, sensori-motrice et affective) étaye le processus d'intersubjectivité primaire chez le sujet (étayage de l'accordage affectif et de la réciprocité des échanges intercorporels) de manière stable et sécurisante. Ce cadre peut ainsi favoriser la possibilité d'émergences au niveau relationnel comme individuel et, notamment, permettre au sujet de prendre des initiatives. En ce sens, notre approche peut contribuer à étayer le mécanisme d'épigénèse interactionnelle, mentionné précédemment.

\section{Prises en charge individuelles non directives standardisées}

Celles-ci ont été conduites en référence à Snoezelen. Notre démarche, combinant méthode clinique et expérimentale, se rapproche de celles exposées par Pernon et Rattaz (2003), Pernon, Pry et Baghdadli (2007) ou Kloekner et coll. (2009). L'objectif de notre démarche est double : $1^{\circ}$ obtenir un impact clinique de la situation elle-même sur le sujet (situation de rencontre, non directivité, succession des séances) et favoriser le bien-être de celui-ci, ce qui offre la possibilité de réaliser des observations en contexte dans le respect du sujet et de l'éthique ; $2^{\circ}$ évaluer la corporéité du sujet dans des situations prédéterminées (par analyse vidéo rétrospective), à l'aide d'une grille d'observation structurée, pouvant objectiver le fonctionnement corporel de la personne en situation d'observation. La situation d'observation est ainsi définie à la base comme non directive, centrée sur la personne, conduite dans le respect du rythme de celle-ci ; elle vise au bien-être et implique la non transgression des refus éventuels, par le sujet, des situations qui lui sont proposées. Les observations ont été réalisées dans une salle spécifiquement aménagée dans l'établissement à cet effet ; l'espace a été structuré de manière minimale, afin de contrôler au maximum l'impact de l'environnement sensoriel.

La structure proprement dite d'une séance de prise en charge se compose de neuf étapes comportant la proposition de prise en charge et le début de séance (étapes A, B, C) ; la ligne de base, correspondant à l'observation initiale du sujet sans action 
de l'intervenant ni modification de l'environnement sensoriel (étape D : contexte sensoriel fixe - silence, lumière fixe - et intersubjectivité minimale de notre part - regard et position par le côté) ; situation Snoezelen (étape E) ; propositions sensorielles prédéfinies et phases de transitions (étape F) ; fin de séance et retour sur le groupe de vie (étapes G, H et I).

L'élaboration des différentes propositions sensorielles (ou items) se rapporte en grande partie à la méthodologie du bilan sensorimoteur (pour enfants) développée par Bullinger (2004). Notre objectif principal était l'investigation clinique et expérimentale de la corporéité des sujets, en testant, notamment de manière écologique et prospective, plusieurs hypothèses relatives au syndrome autistique : «tactilisation » des sens (voir Segond, Maris, Desnos, Bellusso, 2011), difficulté à percevoir les stimuli en mouvement (surtout les stimuli rapides et biologiques), difficultés à percevoir différents types de stimuli sociaux. Nous avons ainsi cherché à explorer la corporéité des sujets principalement selon les différentes dimensions sensorielles impliquées et suivant le caractère social ou non social des stimulations (voir annexe 1. Tableau descriptif des propositions sensorielles et de leurs modalités ${ }^{5}$ ). Ces propositions sensorielles prédéfinies ont été présentées durant les séances en alternance avec des «phases de transition », de durée variable selon les besoins spécifiques à chaque personne. Ceci nous a permis d'associer la dimension éthique, propre à la prise en charge (bientraitance du sujet, respect de ses choix et rythmes), avec le caractère semi-expérimental des séances, ayant pour objectif d'évaluer la corporéité des sujets selon certaines modifications préétablies des flux sensoriels et de la relation intersubjective en situation. Cette méthodologie pose ainsi le primat de la dimension clinique sur le caractère expérimental des situations ; aucun item sensoriel n'a été proposé si le sujet en situation manifestait un signe de refus ou si nous pouvions estimer la situation comme source possible de malêtre ou d'angoisse pour celui-ci.

Pour chacun des sujets, deux sessions de quatre séances chacune ont été conduites ; la seconde session a consisté en une présentation contrebalancée des différentes propositions sensorielles, lorsque cela était possible sur le plan clinique. La durée des séances a été variable selon l'état des sujets (grande variabilité du niveau de fatigue selon les jours) et était généralement comprise entre 60

5. Annexe accessible à cette adresse : http://lpc.unistra. fr/membres/enseignants-chercheurs-titulaires/segond-herve/ et 90 minutes. La fin de séance consistait en une période de relaxation (musique douce), en suite de laquelle nous proposions au sujet de rejoindre son groupe de vie. Comme pour l'entrée, la sortie de la salle a été ritualisée (le sujet était invité à s'asseoir et à remettre ses chaussures) ; le déplacement jusqu'au groupe de vie a été effectué en portant attention aux besoins éventuels de transition.

\section{Grille d'observation structurée}

L'orientation théorique sous-tendant la constitution de notre grille d'observation structurée se réfère aux notions de corporéité et d'intersubjectivité exposées précédemment. Les comportements évalués sont répartis en plusieurs catégories principales, pouvant refléter la corporéité des sujets AA-DIP, dans les différentes situations rencontrées (voir annexe 2. Grille d'observation standardisée des comportements du sujet en situation de prise en charge semi-structurée ${ }^{6}$ ) : utilisation intentionnelle du regard (regard fovéal ou périphérique, envers l'intervenant ou la stimulation présentée), comportements intersubjectifs manifestes initiés (intersubjectivité primaire ou secondaire), manifestations émotionnelles manifestes (à valence positive uniquement, les manifestations émotionnelles négatives entraînant dans tous les cas l'arrêt de la séquence), comportements sensorimoteurs stéréotypés/expressions corporelles autocentrées (permettant d'analyser les stratégies corporelles d'autostimulation ou de pare-excitation sensorielle), et actions sur stimulus-cible (lorsque la situation s'y prête).

\section{Analyse des séquences Ligne de base, Snoezelen et items sensoriels}

Nous avons procédé à une analyse qualitative et quantitative de chaque séquence. Sur le plan quantitatif, nous avons privilégié l'analyse de l'occurrence des comportements selon leurs durées respectives, ceci nous permettant d'établir la proportion temporelle d'occurrence des différents comportements selon les situations. Les situations Ligne de base et Snoezelen ont été également analysées sur le plan chronologique. Les résultats obtenus, pour les différents items sensoriels, nous ont permis une comparaison de la prévalence des différents comportements sensorimoteurs stéréotypés et des manifestations émotionnelles positives selon les modalités sensorielles impliquées et selon le caractère social ou non social des stimulations présentées.

6. Annexe accessible à cette adresse : http://lpc.unistra. $\mathrm{fr} /$ membres/enseignants-chercheurs-titulaires/segond-herve/ 


\section{Enregistrement vidéo}

Nous avons eu recours à l'enregistrement vidéo lors des prises en charges individuelles, parfois même en situation de groupe. Le grand avantage de l'enregistrement vidéo est son pouvoir d'objectivation $a$ posteriori de situations trop complexes pour pouvoir être analysées en contexte. Analyser rétrospectivement via la vidéo des situations de prise en charge individuelles peut permettre une analyse critique plus détaillée de nos comportements envers les sujets, en réduisant notamment l'impact émotionnel ressenti en contexte, ainsi que la mise en évidence des comportements ou séquences de comportements des sujets difficilement perceptibles en situation. Enfin, l'enregistrement vidéo peut faire office de preuve ou d'argument pouvant étayer certaines hypothèses avancées relatives à la problématique des sujets. Si l'utilité de la vidéo est évidente dans notre cadre d'étude, son pouvoir d'objectivation a ses limites. Il faut ainsi souligner que, en référence à notre démarche épistémologique, nous n'avons cessé de considérer que les fondements de notre compréhension de la problématique des sujets reposaient en premier lieu sur notre propre corporéité en contexte, impliquant notre ressenti et compréhension de la corporéité des sujets en situation, du fait du principe d'intercorporéité exposé précédemment. De même, l'analyse vidéo a posteriori du comportement des sujets a inévitablement reposé sur notre propre subjectivité et expérience corporelle, y compris lors de l'utilisation de grilles d'observation standardisées. Cette idée nous semble adéquatement retranscrite par la notion de « corporéité des connaissances » (Penelaud, 2010).

\section{Illustration du profil corporel par une étude de cas}

\section{Anamnèse de Sylvie}

Sylvie est née à terme après une grossesse normale, le 25 décembre 1982 ; son score d'Apgar est de 10 , une minute après sa naissance. Bien qu'un examen pédiatrique, réalisé à ses 9 mois, n'ait rien révélé de suspect, son développement psychomoteur apparaît décalé dès les premières acquisitions, alertant ses parents avant l'âge d'un an. Sylvie est alors décrite comme une enfant anormalement calme, qui pleure très peu ; elle acquiert la marche vers 21 mois, mais avec de nombreuses chutes. En 1984, un bilan, réalisé avec un scanner cérébral, conduit à poser un diagnostic de microcéphalie, sans pouvoir expliquer son origine. Elle est hospitalisée pour retard psychomoteur courant 1985, puis en 1988 pour un traumatisme crânien sans perte de connaissance. Ce n'est qu'en 1994 qu'est posé un diagnostic d'autisme sévèrement déficitaire, avec déficience intellectuelle profonde, par la psychologue de la structure médico-sociale prenant alors Sylvie en charge. En 1996, un nouveau bilan met en évidence, chez elle, un trouble central de la coordination prédominant à droite (suspicion d'hémiparésie secondaire ou primaire), une absence de latéralisation franche dans l'utilisation fonctionnelle de ses membres supérieurs, un steppage assez prononcé au niveau du pied droit (prise en charge orthopédique) et une attitude scoliotique. Enfin, un examen par IRM, réalisé en 1997, met en évidence une très discrète dilatation ventriculaire de nature atrophique probable avec microcéphalie, une hypoplasie du vermis inférieur et une discrète atrophie vermienne. Sylvie a fréquenté une école maternelle au courant de l'année scolaire 1987-1988. Sylvie a été prise en charge à partir de 1989 en institut médico-pédagogique. Elle intègre ensuite un institut médico-éducatif de la Fondation Sonnenhof en 1994, puis le FAM Pierre Valdo à son ouverture en 2004. Sylvie a eu des comportements hétéro-agressifs à plusieurs reprises au cours de son adolescence, notamment envers sa mère. On relève également une période d'un an (2005-2006) pendant laquelle elle a eu un comportement ambivalent envers un autre résident de son groupe de vie : elle cherchait alors très fréquemment le contact physique avec ce dernier et le mordait régulièrement. Actuellement, ses comportements hétéro-agressifs sont très rares. Depuis le décès de son père, il lui arrive parfois de se mordre elle-même. Actuellement, Sylvie est prise en charge par sa mère tous les quinze jours. Elle a, pour seul traitement médicamenteux régulier, un laxatif, ainsi qu'un antispasmodique dès l'apparition des règles.

\section{Résultats diagnostics et aux évaluations} psychologiques

Le tableau 1 présente la synthèse des résultats diagnostics et aux évaluations psychologiques pour Sylvie, alors âgée de 30 ans.

\section{- CARS-T}

Les deux évaluations par l'échelle CARS-T, effectuées auprès de la mère de Sylvie et auprès de son éducateur référent (ER), présentent des résultats homogènes, situant toutes deux Sylvie dans la catégorie de l'autisme sévère. Plusieurs éléments significatifs sont mis en évidence.

Relations sociales : le comportement de Sylvie est très variable ; elle peut ne pas réagir du tout lors d'interactions sociales, comme elle peut réagir 


\begin{tabular}{|c|c|c|}
\hline \multicolumn{3}{|c|}{ SYLVIE (sexe féminin, 30 ans) } \\
\hline CIM-10 & \multicolumn{2}{|c|}{ Autisme infantile (F84.0) et retard mental profond (F73) } \\
\hline \multirow[t]{4}{*}{ CARS-T } & Éducateur référent & Score : 44,5 \\
\hline & & Autisme sévère \\
\hline & Mère & Score : 43 \\
\hline & & Autisme sévère \\
\hline \multirow[t]{8}{*}{ Vineland } & Éducateur référent & - Domaine autonomie / vie quotidienne : 1an 8 mois \\
\hline & & - Domaine motricité : 1 an 4 mois \\
\hline & & - Domaine socialisation : 9 mois \\
\hline & & - Domaine communication : 2 mois \\
\hline & Mère & - Domaine autonomie / vie quotidienne : 1 an 10 mois \\
\hline & & - Domaine motricité : 1 an 9 mois \\
\hline & & - Domaine socialisation : 1 an 2 mois \\
\hline & & - Domaine communication : 10 mois \\
\hline \multirow[t]{8}{*}{ PEP-R } & & Âge de développement global : 6 mois \\
\hline & & - Imitation : épreuves échouées \\
\hline & & - Perception (distale) : âge développemental de 7 mois \\
\hline & & - Motricité fine et coordination oculo-manuelle : épreuves \\
\hline & & échouées \\
\hline & & - Motricité globale : âge développemental de 1 an \\
\hline & & - Performances cognitives : épreuves échouées \\
\hline & & - Cognition verbale : pas de langage verbal fonctionnel \\
\hline
\end{tabular}

Tableau 1. Synthèse des résultats diagnostics et aux évaluations psychologiques pour Sylvie.

immédiatement à la présence et au comportement d'autrui. Elle apparaît, de plus, présenter des troubles majeurs de la régulation émotionnelle : elle peut passer du rire aux larmes et inversement, sans rapport évident avec la situation, bien que ces réactions émotionnelles semblent souvent induites par des stimuli auditifs (musique et ton de la voix). Sylvie présente également des réactions de peur excessives et inappropriées aux situations.

Utilisation du corps : elle est atypique : Sylvie reste le plus souvent statique (pas de déplacement), elle semble présenter un trouble de la coordination quant à l'utilisation de ses membres et elle présente de fréquentes stéréotypies sensorimotrices (en particulier, un balancement du buste). Elle présente peu d'intérêt pour les objets ou les jouets, excepté pour les livres d'images.

Fonctionnement visuel : généralement atypique : Sylvie a fréquemment un regard fixe « dans le vide », évite souvent de croiser le regard d'autrui ; en outre, elle ne regarde généralement pas ses mains lorsqu'elle effectue des actions.

Domaine auditif : la mère de Sylvie comme son ER soulignent tous deux une hyperréactivité chez elle à la musique et au ton de la voix, manifestant parfois des réactions hétéroagressives à la voix.
Domaine gustatif : Sylvie présente une certaine sélectivité quant aux aliments et à la boisson.

Domaine tactile : on relève, d'une part, une hyporéactivité à la douleur (surtout durant son enfance et son adolescence) et, d'autre part, une appétence variable selon les moments pour les contacts tactiles (massages, exploration tactile d'autrui et de l'environnement).

Compétences communicatives : Sylvie ne possède pas de langage verbal fonctionnel, mais répète parfois des syllabes ou ce qui semble être des mots ou des noms de personnes. Les avis sur ses compétences de communication non verbale divergent : selon sa mère, Sylvie est capable de pointer (du bras et de la main), de signifier un besoin en touchant autrui du doigt, ainsi que de répondre aux sourires qui lui sont adressés. L'ER de Sylvie n'observe, quant à lui, pas de communication intentionnelle de sa part.

Aptitude à s'adapter aux changements : les avis divergent également. Selon sa mère, Sylvie résiste activement aux changements de routine (maintien de ses propres activités tout en cherchant à rester en contact) ; selon son ER, Sylvie ne manifeste jamais de réaction à ces changements dans son groupe de vie. 


\section{- Vineland}

Les deux évaluations par les échelles Vineland, effectuées auprès de la mère de Sylvie et auprès de son ER, présentent des résultats différents, tout en offrant une comparaison intéressante : en effet, la hiérarchisation entre les différents domaines évalués, selon leur score respectif, est identique (même profil), alors que, pour chacun de ces domaines, les résultats obtenus auprès de la mère de Sylvie sont, à chaque fois, légèrement supérieurs à ceux obtenus auprès de son ER.

Autonomie dans la vie quotidienne : c'est dans ce domaine que l'on relève les meilleurs scores. Selon les deux évaluations, Sylvie paraît pouvoir s'alimenter (en utilisant une cuillère ou une fourchette) et boire sans aide ; elle comprend également que ce qui est chaud est dangereux. Elle a besoin d'être guidée pour se vêtir ou se dévêtir. Bien qu'elle puisse utiliser les toilettes de manière fonctionnelle, elle reste incontinente de jour comme de nuit. Selon sa mère (et non selon son ER), Sylvie manifeste occasionnellement son besoin d'aller aux toilettes ou de changer de vêtements, lorsque ceux-ci sont mouillés ou sales (par rapprochement physique et contact tactile) ; elle peut également ranger ses affaires sur demande, ainsi qu'aider occasionnellement à certains travaux ménagers.

Domaine de la motricité : Sylvie se déplace en marchant sans difficulté manifeste, elle peut monter et descendre des escaliers, ainsi que s'asseoir et adopter la position couchée de manière autonome. Elle est occasionnellement capable de courir. Elle ne semble pas pouvoir se déplacer lorsqu'elle est accroupie. Elle utilise ses mains fonctionnellement (transfert manuel, prise digitale) de manière habituelle selon sa mère, seulement quelquefois ou en partie selon son ER. Elle peut tenir un crayon pour gribouiller. Selon sa mère (et non selon son ER), elle peut ouvrir les portes en manipulant les poignées, dévisser parfois des couvercles (sur demande), ainsi que sauter sur place en s'aidant d'un appui. Selon son ER, Sylvie est parfois capable de conduire un tricycle.

Domaine de la socialisation : selon l'ER, ses capacités sont extrêmement limitées ; Sylvie manifeste de manière reconnaissable son bien-être et son malêtre, réagit occasionnellement à la présence d'autrui, fait parfois preuve d'intérêt envers de nouvelles personnes ou envers les activités de personnes familières, joue uniquement seule et de manière stéréotypée avec certains objets, peut parfois faire preuve d'affection, ainsi que réagir occasionnellement de manière positive à des compliments. L'ER de Sylvie ne sait pas si celle-ci différencie véritablement les personnes en présence. Selon sa mère, tout d'abord, ces capacités ne s'expriment pas occasionnellement, mais de manière habituelle chez elle. De plus, outre le fait que Sylvie identifie les personnes en présence, elle marque clairement ses préférences pour certaines personnes et aime jouer à des jeux interactifs simples avec autrui ; elle peut également imiter parfois des gestes simples. La mère de Sylvie, comme son ER, notent tous deux l'absence de jeu symbolique chez elle.

Domaine de la communication : selon l'ER de Sylvie, ses compétences sont quasi inexistantes et se limitent à écouter occasionnellement la personne qui lui parle, à sourire parfois à la présence de certaines personnes et à manifester occasionnellement sa compréhension du «non ». Selon sa mère, le fait que Sylvie écoute ou non la personne qui s'adresse à elle dépend du «chanté » de la voix (rythme et tonalité) ; de même, sa compréhension du « non » dépend du ton employé. Ses compétences communicatives (réceptives et expressives) semblent variables selon les moments et les situations : en certaines occasions, elle manifeste sa compréhension d'au moins dix mots de vocabulaire, peut parfois suivre certaines instructions faisant intervenir une action ou un objet, peut parfois montrer du doigt une partie de son corps quand on lui demande, ainsi que nommer par leur nom ou diminutif certaines des personnes familières de son entourage. Elle peut imiter certains sons immédiatement après les avoir entendus. Quelquefois, elle peut manifester des mimiques et gestes appropriés pour indiquer son refus ou un besoin. Elle ne semble pas comprendre le sens de « oui » ou de «d'accord », ni indiquer une préférence lorsqu'on lui propose un choix. Elle ne possède pas de langage verbal fonctionnel.

\section{- PEP-R}

L'évaluation de Sylvie par le PEP-R permet de dresser son profil développemental en mettant en évidence ses réussites, ses échecs et ses capacités en émergence dans sept domaines de développement. Nous reprendrons ici les résultats principaux de cette évaluation.

Domaine de l'imitation : Sylvie échoue à toutes les épreuves (âge développemental non calculable). Elle n'imite pas de manière spontanée et échoue à imiter sur demande, qu'il s'agisse de gestes simples, de manipulation d'objets, de sons ou de mots, bien que l'on observe des capacités émergentes à pouvoir imiter des actions impliquant des objets sonores (Sylvie manifeste un intérêt certain pour de tels 
objets). En revanche, elle paraît sensible à l'imitation de ses propres sons (babillage spontané).

Domaine de la perception (distale) : au niveau auditif, elle s'oriente vers la source des sons émis avec des objets comme un sifflet, une clochette ou des claquettes. Sa perception visuelle est atypique, voire déficitaire : on note une émergence à suivre du regard des objets en mouvement lent, comme une bulle de savon, mais on observe de manière générale un désengagement fréquent du regard et une absence de latéralisation oculaire. Sans que l'on puisse définir si ces troubles sont davantage relatifs à son fonctionnement visuel per se ou à ses capacités de compréhension, Sylvie ne réagit pas à des gestes simples (comme « donne-moi » ou « viens ») et n'apparaît pas capable de reconnaître ou d'associer des objets d'après leur forme, taille ou couleur.

Domaine de la motricité fine et de la coordination oculo-manuelle : Sylvie échoue à toutes les épreuves (âge développemental non calculable). Elle ne regarde presque jamais ses mains durant ses actions et la question de sa compréhension des activités elles-mêmes se pose. On observe cependant certaines capacités émergentes dans ce domaine. Elle peut manipuler certains objets fins ou petits (bâtonnet, perles en bois, crayon), mais sans pince digitale adéquate (elle prend les objets à pleine main). Elle échoue systématiquement à toute activité impliquant une construction ou une élaboration de forme.

Motricité globale : ce domaine est celui dans lequel Sylvie obtient ses meilleures performances. Elle marche et s'assied de manière autonome, peut lancer un ballon et utiliser un verre de manière fonctionnelle. Elle transfère quelquefois des objets d'une main à l'autre, mais semble présenter un trouble de la latéralisation manuelle. De même, elle apparaît présenter un trouble de la latéralisation du pied.

Domaine des performances cognitives : Sylvie échoue à toutes les épreuves (âge développemental non calculable). On observe seulement une émergence de ses capacités à retrouver un objet caché devant elle (recherche non spontanée). De même, elle ne manifeste aucune compétence dans le domaine de la cognition verbale : elle n'utilise pas de langage verbal de manière fonctionnelle durant l'évaluation.

Comportement : Sylvie présente une conduite atypique durant la session d'évaluation. Elle manifeste un plaisir certain pour cette situation (grands sourires et de nombreux babillages à tonalité joyeuse), mais le matériel présenté ne suscite, en général, chez elle qu'un intérêt très modéré ; de plus, elle ne semble pas comprendre les demandes et le sens des activités proposées, malgré un guidage important. Lorsqu'elle tente de répondre à la demande de l'examinateur, on observe chez elle une capacité d'attention très limitée. Ses réponses aux stimuli sensoriels mettent en évidence une hypersensibilité aux sons, un évitement inhabituel des stimuli visuels (ses explorations visuelles des objets ne durent que quelques secondes), mais une sensibilité tactile, olfactive et gustative apparemment normale. Sylvie manifeste tout du long beaucoup de stéréotypies sensorimotrices, mais de manière assez légère : on observe des balancements du buste, des mouvements soudains de la tête, des mouvements atypiques des mains et des doigts, ainsi que des contractions soudaines des muscles du visage (paupières et bouche principalement). Elle semble, en fait, principalement intéressée par l'interaction sociale avec l'examinateur : on observe chez elle une forte réactivité à la tonalité de la voix de ce dernier, et de fréquents, mais courts, regards dirigés ; elle initie même plusieurs interactions sociales à l'endroit de l'examinateur par de légers contacts tactiles avec la main. Il nous apparaît ainsi clairement que Sylvie se situe principalement à un niveau d'intersubjectivité primaire (communication intercorporelle immédiate).

\section{Résultats des questionnaires d'auto-évaluation} de la prise en charge de Sylvie

- Dimension socio-affective du cadre d'accompagnement de Sylvie

$\mathrm{Au}$ moment de notre investigation, Sylvie est prise en charge au sein d'un groupe de vie constitué de dix résidents et huit accompagnants. Les résultats aux questionnaires d'auto-évaluation mettent en évidence un accompagnement au quotidien de Sylvie de difficulté très variable pour les accompagnants du groupe. Les difficultés les plus manifestes sont la capacité des accompagnants à se faire comprendre de Sylvie, ainsi qu'à comprendre ses demandes, avec, cependant, une forte variabilité selon les accompagnants. Les réponses à la question « à quel degré estimez-vous connaître Sylvie ?» révèlent également une très grande variabilité selon les accompagnants.

\section{- Corporéité de Sylvie au quotidien}

Expressivité émotionnelle : les modalités d'expression des états de bien-être ou de plaisir de Sylvie apparaissent bien identifiées par ses accompagnants. L'expression du visage (muscles détendus et sourire) constitue le marqueur le plus 
cité, suivi par les mouvements du corps (balancements proches de la danse, généralement en présence de musique). Les accompagnants relèvent également, dans ce contexte, les expressions vocales de Sylvie (rires et exclamations ou babillage à tonalité joyeuse), son utilisation du regard (yeux décrits comme «pétillants »; regards dirigés vers autrui plus soutenus et plus fréquents), ainsi que son utilisation du toucher envers autrui (contacts tactiles plus fréquents et plus soutenus, parfois sous la forme de caresses). Les modalités d'expression des états de mal-être de Sylvie apparaissent encore plus évidentes aux accompagnants : visage crispé, regard décrit comme «fermé » ou « triste », cris et parfois pleurs, mouvements du corps brusques et saccadés (dans les déplacements et les gestes). Certains accompagnants signalent de plus que Sylvie peut parfois se mettre en colère et manifester alors de l'hétéro-agressivité (elle frappe de la main). Selon certains accompagnants, l'état émotionnel de Sylvie semble lié au niveau de stress et d'agitation au niveau du groupe. Néanmoins, plusieurs d'entre eux expriment leur incompréhension fréquente quant aux causes de ses manifestations émotionnelles négatives.

Stratégies de communication intentionnelle : les accompagnants décrivent de manière consensuelle que Sylvie entre intentionnellement en contact avec autrui en se rapprochant physiquement de la personne, en dirigeant son regard vers le visage de l'autre, ainsi qu'en le touchant avec la main ou, parfois, en l'embrassant. L'utilisation de sa voix est également rapportée comme participant à ses stratégies de communication intentionnelle (babillage). Certains accompagnants considèrent que les mouvements du corps de Sylvie (balancements) peuvent être émis intentionnellement par elle pour étayer la relation intersubjective.

Latéralité manuelle : une forte majorité des accompagnants considère que Sylvie utilise préférentiellement sa main droite.

Recherche active de sensations : les accompagnants de Sylvie observent, de manière consensuelle, que les principales parties du corps qu'elle stimule sont ses mains, ses organes génitaux, son crâne/sa tête et son anus. Quant aux modalités sensorielles investies par Sylvie, ils rapportent sa très forte sensibilité envers les stimuli auditifs et spécifiquement pour la musique (vocale ou instrumentale). De nombreuses remarques mettent en évidence que l'écoute de musique déclenche chez elle de fortes réactions émotionnelles, selon le type de rythme et de mélodie : des musiques au rythme soutenu et à tonalité « joyeuse » semblent entraîner chez elle des manifestations émotionnelles positives et une excitation corporelle importante (balancements et mouvements des bras et des mains), alors que des musiques au rythme lent et à tonalité « triste » semblent entraîner chez elle des manifestations émotionnelles négatives (expression de tristesse manifeste, allant parfois jusqu'aux pleurs) et un ralentissement de l'activité corporelle. Les accompagnants rapportent également, de manière consensuelle, que Sylvie recherche fréquemment des sensations tactiles fines, principalement au moyen de ses doigts, en se caressant ou se frottant le corps (notamment la tête) ou en explorant les objets ou les personnes dans son environnement immédiat. Il est également fait état de sa recherche de sensations vibratoires au niveau des mains, qu'elle frappe fréquemment entre elles comme pour applaudir. De manière non consensuelle, plusieurs accompagnants rapportent de plus que Sylvie recherche des stimulations kinesthésiques et vestibulaires (principalement par mouvements des bras et par balancements du buste), des stimulations olfactives et gustatives, des stimulations visuelles, ainsi que des sensations de pression. Il est intéressant de remarquer qu'il apparaît généralement difficile, pour les accompagnants, de préciser et d'affiner leurs réponses à ces questions spécifiques.

Étude de la corporéité de Sylvie par analyse des situations de prise en charge individuelles non directives semi-standardisées : analyse globale des résultats

Nous avons effectué deux sessions, constituées chacune de quatre séances d'une heure environ, réparties, à chaque fois, sur une semaine. La seconde session a eu lieu environ trois mois après la première, du fait du décès du père de Sylvie à cette période, puis de sa prise en charge par sa mère autour de la période des fêtes de fin d'année.

Globalement, Sylvie a réagi de manière particulièrement positive à chaque séance de prise en charge. Elle a manifesté très fréquemment son bien-être par de nombreux sourires, ainsi que par des rires et des exclamations à tonalité joyeuse (traduisant selon nous son plaisir ou son amusement). Nous n'avons observé aucune manifestation émotionnelle négative au cours des séances.

Le toucher revêt une importance toute particulière dans les rapports de Sylvie avec son environnement, en particulier à travers ses nombreux comportements intersubjectifs intentionnels, manifestés durant les séances, mais, également, au niveau de ses compor- 
tements sensorimoteurs stéréotypés. Nous avons ainsi relevé une grande utilisation du regard fovéal vers l'intervenant, ainsi que de fréquentes recherches de contact tactile manuel envers l'intervenant, sous différentes formes : contact tactile fin sur les mains, les avant-bras ou le visage de l'intervenant ; contact tactile par pression en serrant les mains ou en entourant la nuque de l'intervenant ; contact tactile par pression au niveau des mains de l'intervenant, associé à un balancement rythmé et dirigé ; contact tactile vibratoire en frappant des paumes celles de l'intervenant. À plusieurs reprises, Sylvie a également tendu à l'intervenant un objet qui lui avait été présenté, manifestant ainsi des capacités relatives au niveau de l'intersubjectivité secondaire. Du fait, des manifestations émotionnelles positives contingentes à ces situations, ces comportements de don d'objets ont été interprétés comme des demandes d'interaction autour de l'objet.

$\mathrm{Au}$ niveau de ses stéréotypies, nous relevons, tout d'abord, des comportements d'autostimulation tactilo-kinesthésique des bras ou des mains : ceux-ci correspondent, soit à des mouvements bien visibles des bras et des mains, aboutissant parfois à une autostimulation tactile du crâne ou du visage, soit à des mouvements plus discrets, mais plus fréquents, des doigts, frottés doucement les uns contre les autres, les mains étant jointes de manière atypique. Nous relevons ensuite de fréquents mouvements atypiques de la bouche chez Sylvie, consistant en une ouverture prononcée, puis une fermeture des lèvres, souvent similaire à une grimace de dégoût chez un sujet tout-venant. Cependant, nos observations en situation nous ont amenés à écarter l'hypothèse que ce comportement soit lié à l'expression d'un vécu à valence négative de ce type. Sylvie a également manifesté régulièrement des comportements d'autostimulation tactilo-kinesthésique des jambes ou des pieds, souvent de manière rythmique. Nous avons également observé, chez Sylvie, de fréquents balancements du corps, sur le plan latéral ou le plan sagittal. Nous avons également relevé, chez Sylvie, des comportements assez fréquents de clignement prononcé des yeux et de mouvement brusque de la tête vers l'arrière, ces comportements étant parfois combinés avec un mouvement atypique de la bouche. Nous relevons de plus, occasionnellement, chez Sylvie, un comportement spécifique d'agitation de ses mains et de ses doigts devant ses yeux. Enfin, nous notons la présence peu fréquente d'un comportement de contraction musculaire soudaine du buste/sursaut tonique.
- Comparaisons situations Ligne de base (LB) et Snoezelen (SNZ; propositions sensorielles)

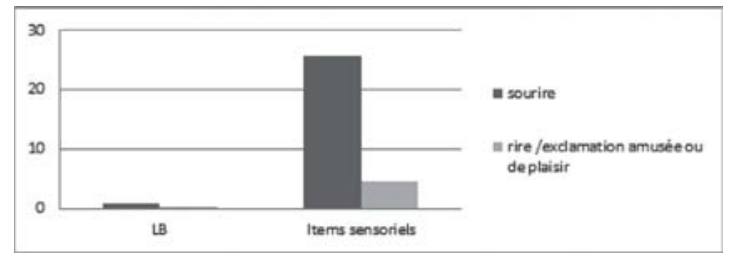

Graphique 1. Proportion temporelle de l'occurrence des manifestations émotionnelles.

\begin{tabular}{lcc}
\hline & Ligne de base & Items sensoriels \\
\hline Sourire & 0,83 & 25,73 \\
\hline $\begin{array}{l}\text { Rire/exclama- } \\
\text { tion amusée }\end{array}$ & 0,07 & 4,55 \\
ou de plaisir & & \\
\hline
\end{tabular}

Tableau 3. Proportion temporelle de l'occurrence des manifestations émotionnelles.

La comparaison entre situations LB et SNZ révèle qu'en l'absence de modification extérieure des flux sensoriels (LB), Sylvie s'autostimule régulièrement par plusieurs stéréotypies sensorimotrices (graphique 2 et tableau 4). Elle entretient, de ce fait, un niveau minimal et continu de stimulation, en générant d'elle-même des modifications du flux tactile (surtout au niveau des doigts, des mains et des bras), du flux visuel, ainsi que du flux vestibulaire (en proportion moindre).

La prévalence de l'ensemble des comportements sensorimoteurs stéréotypés (indice général) augmente dans les situations de propositions sensorielles (graphique 2 et tableau 4), tout comme les manifestations émotionnelles positives (graphique 1 et tableau 3). Cependant, on relève des différences quant aux modifications des prévalences respectives des différentes stéréotypies sensorimotrices analysées.

Tout d'abord, le comportement d'agitation des mains/doigts devant les yeux est le seul dont la prévalence diminue dans les situations de propositions sensorielles : la fonction de ce comportement semble ainsi directement liée à un besoin de stimulation visuelle. On relève ensuite l'augmentation manifeste de la prévalence des comportements suivants : mouvement atypique des lèvres, mouvement des pieds et des jambes, balancement du buste, mouvement brusque et prononcé de la tête, et fermeture prononcée des paupières. Ces comportements semblent ainsi participer 
de manière directe à l'autorégulation active des flux sensoriels chez Sylvie lors des situations de propositions sensorielles. On observe enfin que la prévalence du comportement d'autostimulation tactilo-kinesthésique des bras ou des mains (préva- lence la plus forte), tout comme celle du comportement de sursaut tonique du dos et des épaules (prévalence la plus faible), n'augmentent que de manière infime lors des situations de propositions sensorielles.

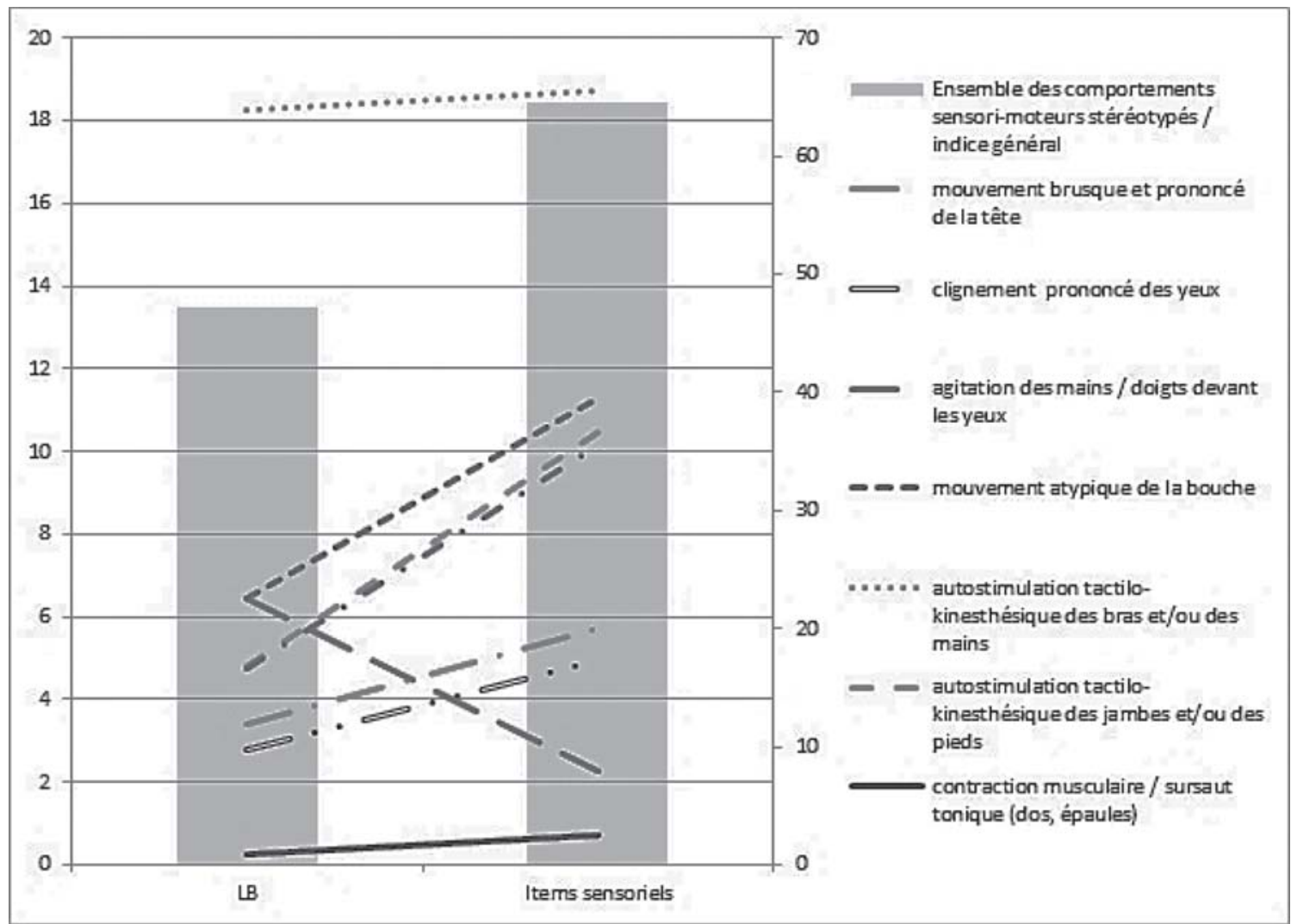

Graphique 2. Proportion temporelle de l'occurrence des principaux comportements sensorimoteurs stéréotypés relevés.

Remarque : l'axe des ordonnées, situé à droite du graphique, correspond à la proportion temporelle de l'occurrence de l'ensemble des comportements sensorimoteurs stéréotypés (indice général).

\begin{tabular}{lcc}
\hline & $\begin{array}{c}\text { Ligne de } \\
\text { base }\end{array}$ & $\begin{array}{c}\text { Items } \\
\text { sensoriels }\end{array}$ \\
\hline $\begin{array}{l}\text { Ensemble des comportements sensorimoteurs } \\
\text { stéréotypés / indice général }\end{array}$ & 47,15 & 64,61 \\
\hline Mouvement brusque et prononcé de la tête & 3,4 & 5,72 \\
\hline Clignement prononcé des yeux & 2,77 & 4,96 \\
\hline Agitation des mains / doigts devant les yeux & 6,46 & 2,25 \\
\hline Mouvement atypique de la bouche & 6,46 & 11,31 \\
\hline $\begin{array}{l}\text { Autostimulation tactilo-kinesthésique des jambes et/ } \\
\text { ou des pieds }\end{array}$ & 18,26 & 18.74 \\
\hline $\begin{array}{l}\text { Contraction musculaire / sursaut tonique (dos, } \\
\text { épaules) }\end{array}$ & 4,79 & 10,48 \\
\hline Balancement du corps & 0,28 & 10,17 \\
\hline
\end{tabular}

Tableau 4. Proportion temporelle de l'occurrence des principaux comportements sensorimoteurs stéréotypés relevés. 
- Comparaison par modalités sensorielles

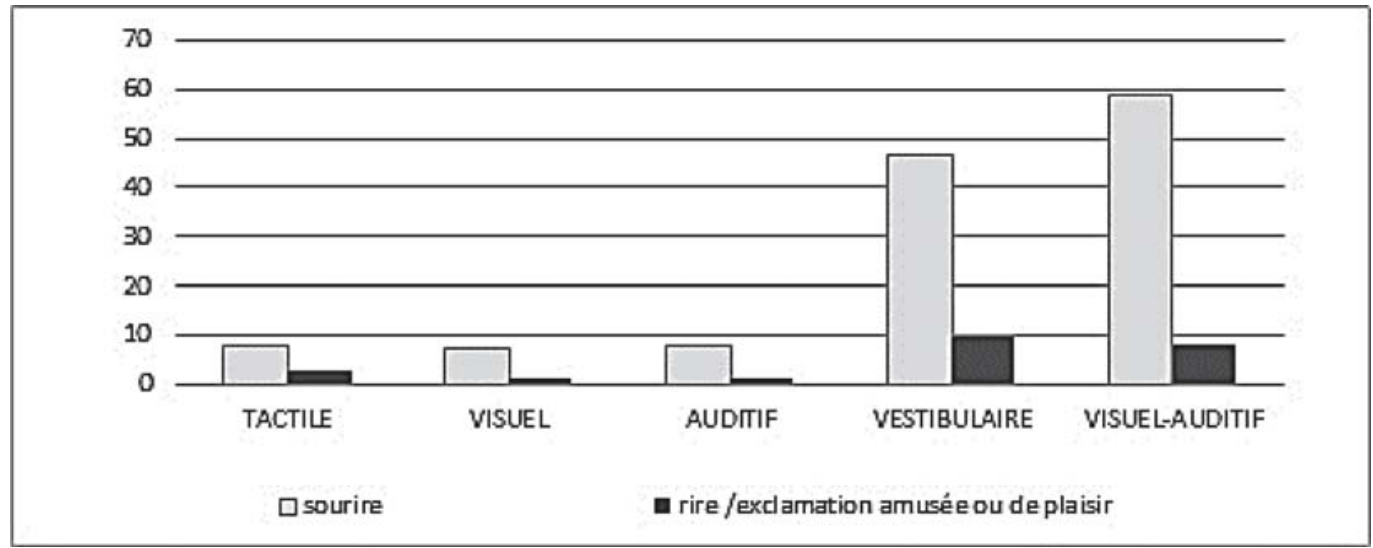

Graphique 3. Proportion temporelle de l'occurrence des manifestations émotionnelles.

\begin{tabular}{lccccc} 
& Tactile & Visuel & Auditif & Vestibulaire & Visuel-auditif \\
\cline { 2 - 6 } Sourire & 7,64 & 7,55 & 8,05 & 46,7 & 58,7 \\
\cline { 2 - 6 } $\begin{array}{l}\text { Rire/exclamation amusée ou } \\
\text { de plaisir }\end{array}$ & 2,69 & 1,15 & 1,02 & 9,89 & 7,96 \\
\hline
\end{tabular}

Tableau 5. Proportion temporelle de l'occurrence des manifestations émotionnelles.

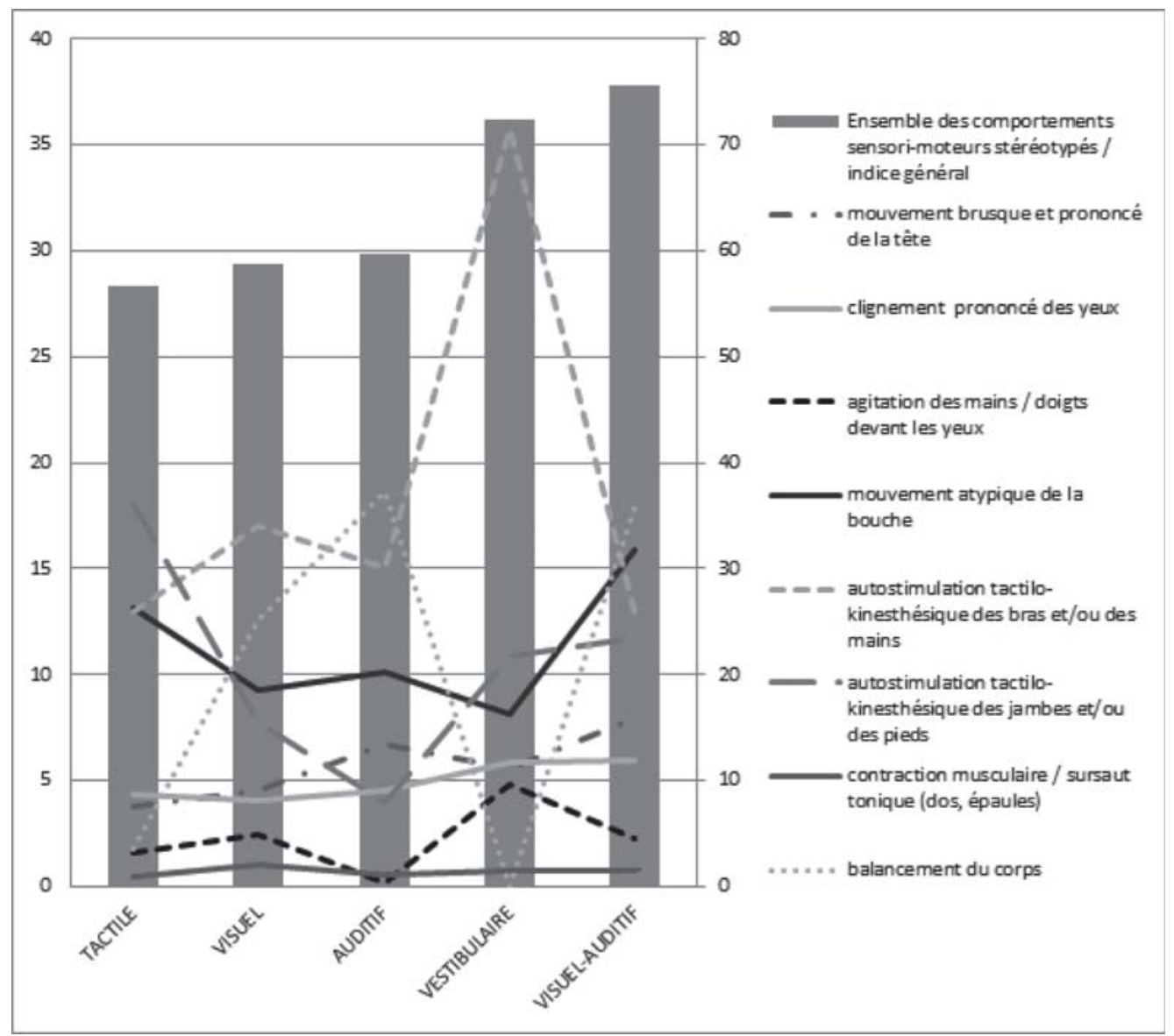

Graphique 4. Proportion temporelle de l'occurrence des comportements sensorimoteurs stéréotypés relevés.

Remarque : l'axe des ordonnées situé à droite du graphique correspond à la proportion temporelle de l'occurrence de l'ensemble des comportements sensorimoteurs stéréotypés (indice général). 


\begin{tabular}{lccccc} 
& Tactile & Visuel & Auditif & $\begin{array}{c}\text { Vesti- } \\
\text { bulaire }\end{array}$ & $\begin{array}{c}\text { Visuel- } \\
\text { auditif }\end{array}$ \\
\cline { 2 - 6 } $\begin{array}{l}\text { Ensembles des comportements sensorimo- } \\
\text { teurs stéréotypés/indice général }\end{array}$ & 56,63 & 58,74 & 59,74 & 72,33 & 75,62 \\
\hline Mouvement brusque et prononcé de la tête & 3,82 & 4,51 & 6,73 & 5,58 & 7,96 \\
\hline Clignement prononcé des yeux & 4,38 & 4,10 & 4,54 & 5,84 & 5,97 \\
\hline Agitation des mains / doigts devant les yeux & 1,57 & 2,46 & 0,15 & 4,82 & 2,24 \\
\hline Mouvement atypique de la bouche & 13,15 & 9,27 & 10,10 & 8,12 & 15,92 \\
\hline $\begin{array}{l}\text { Autostimulation tactilo-kinesthésique des } \\
\text { jambes ou des pieds }\end{array}$ & 18,09 & 7,79 & 3,95 & 10,91 & 11,69 \\
\hline $\begin{array}{l}\text { Contraction musculaire / sursaut tonique } \\
\text { (dos, épaules) }\end{array}$ & 0,45 & 0,98 & 0,58 & 0,76 & 0,75 \\
\hline Balancement du corps & 1,80 & 12,55 & 18,59 & 0 & 17,91 \\
\hline
\end{tabular}

Tableau 6. Proportion temporelle de l'occurrence des comportements sensorimoteurs stéréotypés relevés.

La prévalence de l'ensemble des comportements sensorimoteurs stéréotypés (indice général), les prévalences respectives des stéréotypies sensorimotrices, ainsi que celles relatives aux manifestations émotionnelles varient selon la nature des flux sensoriels modifiés.

Afin de pouvoir affiner nos interprétations du graphique 4 et du tableau 6 , nous avons choisi de synthétiser sous forme graphique le niveau d'importance de modification par Sylvie de ses flux sensoriels, selon la nature des comportements sensorimoteurs stéréotypés relevés.

On observe ainsi que les modifications d'origine externe du flux tactile (modalité « tactile ») sont les situations qui suscitent, chez Sylvie, le moins d'agitation motrice (voir l'indice général du taux de prévalence de l'ensemble des stéréotypies sensorimotrices ; graphique 4 et tableau 6) et, par conséquent, les modifications les moins importantes des différents flux sensoriels (graphique 5 et tableau 7), bien que les modifications du flux tactile restent importantes dans l'ensemble, via l'agitation de ses bras, de ses mains et de ses doigts, de ses jambes et de ses pieds, ainsi que de ses lèvres. Ces situations ne suscitent également, chez elle, que peu de manifestations émotionnelles positives (graphique 3 et tableau 5). Sur le plan clinique, Sylvie apparaît alors plus calme.

À l'inverse, les stimulations extérieures de nature visuo-auditive (modalité « visuel-auditif ») sont les situations qui suscitent chez elle le plus d'agitation motrice (voir l'indice général du taux de prévalence de l'ensemble des stéréotypies sensorimotrices ; graphique 4 et tableau 6) et les modifications les plus importantes de chaque flux sensoriel (graphique 5 et tableau 7). Ce sont également les situations qui entraînent de sa part le plus de sourires, ainsi que plusieurs rires et exclamations amusées (graphique 3 et tableau 5). On relève, dans ces situations, une forte prévalence de certains de ses comportements, respectivement les balancements, le mouvement des lèvres, des doigts/mains/

\begin{tabular}{|c|c|c|c|c|}
\hline Comportement & Flux sen & oriel(s) & difié(s) & \\
\hline $\begin{array}{l}\text { autostimulation tactilo-kinesthésique des bras et/ou des } \\
\text { mains }\end{array}$ & tactile & & & \\
\hline mouvement atywpique de la bouche & tactile & & & \\
\hline $\begin{array}{l}\text { autostimulation tactilo-kinesthésique des jambes et/ou } \\
\text { des pieds }\end{array}$ & tactile & & & \\
\hline balancement du corps & tactile & visuel & vestibulaire & auditif \\
\hline mouvement brusque et prononcé de la tête & tactile & visuel & vestibulaire & auditif \\
\hline clignement prononcé des yeux & tactile & visuel & & \\
\hline agitation des mains / doigts devant les yeux & tactile & visuel & & \\
\hline contraction musculaire / sursaut tonique (dos, épaules) & tactile & & & \\
\hline
\end{tabular}

Tableau 2. Table de correspondance entre les comportements sensorimoteurs stéréotypés observés et les modifications des flux sensoriels impliquées. 


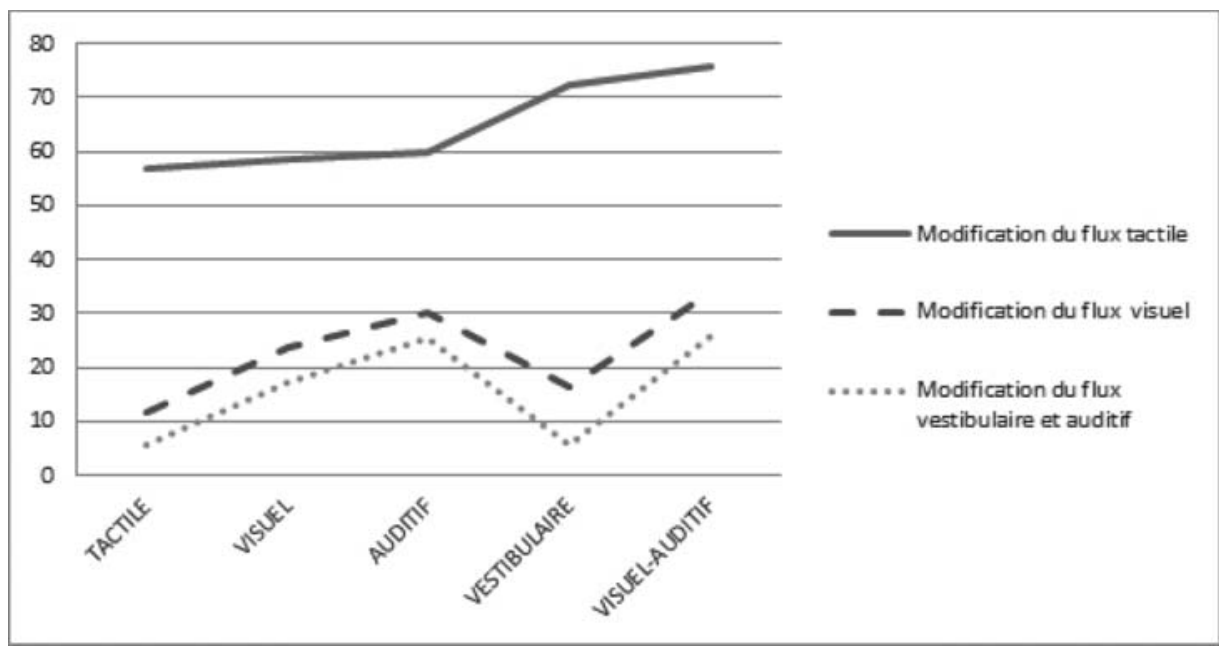

Graphique 5. Indice de modification des flux sensoriels par les stéréotypies sensorimotrices du sujet selon la nature des situations de propositions sensorielles.

\begin{tabular}{lccccc}
\cline { 2 - 6 } & Tactile & Visuel & Auditif & $\begin{array}{c}\text { Vesti- } \\
\text { bulaire }\end{array}$ & Visuel-auditif \\
\hline Modification du flux tactile & 56,63 & 58,74 & 59,74 & 72,33 & 75,62 \\
\hline Modification du flux visuel & 11,57 & 23,63 & 30,01 & 16,24 & 34,07 \\
\hline $\begin{array}{l}\text { Modification du flux vestibulaire } \\
\text { et auditif }\end{array}$ & 5,62 & 17,06 & 25,33 & 5,58 & 25,87 \\
\hline
\end{tabular}

Tableau 7. Indice de modification des flux sensoriels par les stéréotypies sensorimotrices du sujet selon la nature des situations de propositions sensorielles.

bras et des pieds. Les stimulations visuo-auditives présentées sont toutes de nature sociale (elles impliquent directement l'intervenant), mais, de ce fait, elles représentent également le type de stimulations certainement le plus rencontré par Sylvie dans son environnement quotidien (parole adressée, par exemple).

Les stimulations extérieures de nature purement auditive (modalité « auditif »), même si elles suscitent une moindre prévalence de stéréotypies sensorimotrices (voir indice général ; graphique 4 et tableau 6), conduisent également Sylvie à adopter une attitude où prédominent ses comportements de balancement et de mouvement des doigts/mains/ bras. Il apparaît ainsi manifeste que le comportement de balancement est en premier lieu fonctionnellement lié aux modifications externes du flux auditif. On peut, d'ailleurs, remarquer que, de manière similaire, mais bien moins manifeste, les plus fortes prévalences du comportement «mouvement brusque et prononcé de la tête » sont observées lors des stimulations externes de nature visuoauditive ou seulement auditive. L'augmentation de la prévalence de ce comportement semble donc aussi liée aux variations du flux auditif.
Lors des stimulations extérieures de nature purement visuelle (modalité « visuel »; voir graphique 4 et tableau 6), les comportements présentant les plus fortes prévalences sont respectivement le mouvement des doigts/mains/bras en premier lieu, le balancement, le mouvement des lèvres et le mouvement des pieds/jambes. Le balancement apparaît ainsi participer également de manière importante à l'autorégulation par Sylvie de son flux visuel, contrairement au comportement de fermeture prononcée des paupières, dont la prévalence n'augmente pas lors de situations de modification du flux visuel.

Quant aux situations de modification du flux vestibulaire (modalité « vestibulaire »), elles suscitent, chez Sylvie, beaucoup de plaisir (graphique 3 et tableau 5), tout en entraînant une agitation motrice très importante, principalement par des mouvements des mains et des bras (graphique 4 et tableau 6). On remarque aussi que c'est dans cette condition que le comportement d'agitation des mains/doigts présente la plus forte prévalence. Sur le plan clinique, il nous apparaît que, bien qu'elle apprécie beaucoup ce type de stimulation, Sylvie n'est pas totalement à l'aise et utilise ses mains pour intensifier sa perception de ses propres limites corporelles. 
- Comparaison selon le caractère social ou non des propositions sensorielles

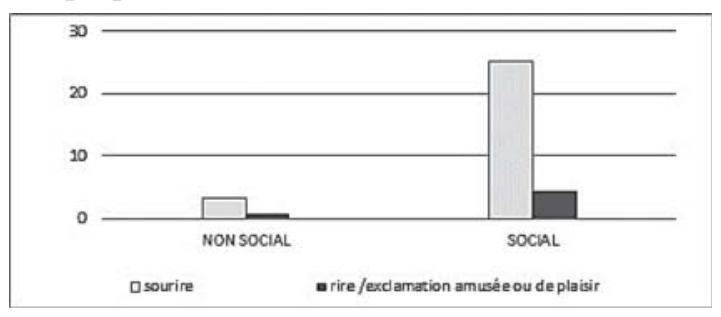

Graphique 6. Proportion temporelle de l'occurrence des manifestations émotionnelles.

\begin{tabular}{lll}
\hline & Non social & Social \\
\hline Sourire & 3,32 & 25,19 \\
\hline $\begin{array}{l}\text { Rire / exclamation } \\
\text { amusée ou de plaisir }\end{array}$ & 0,92 & 4,63 \\
\hline
\end{tabular}

Tableau 8. Proportion temporelle de l'occurrence des manifestations émotionnelles.

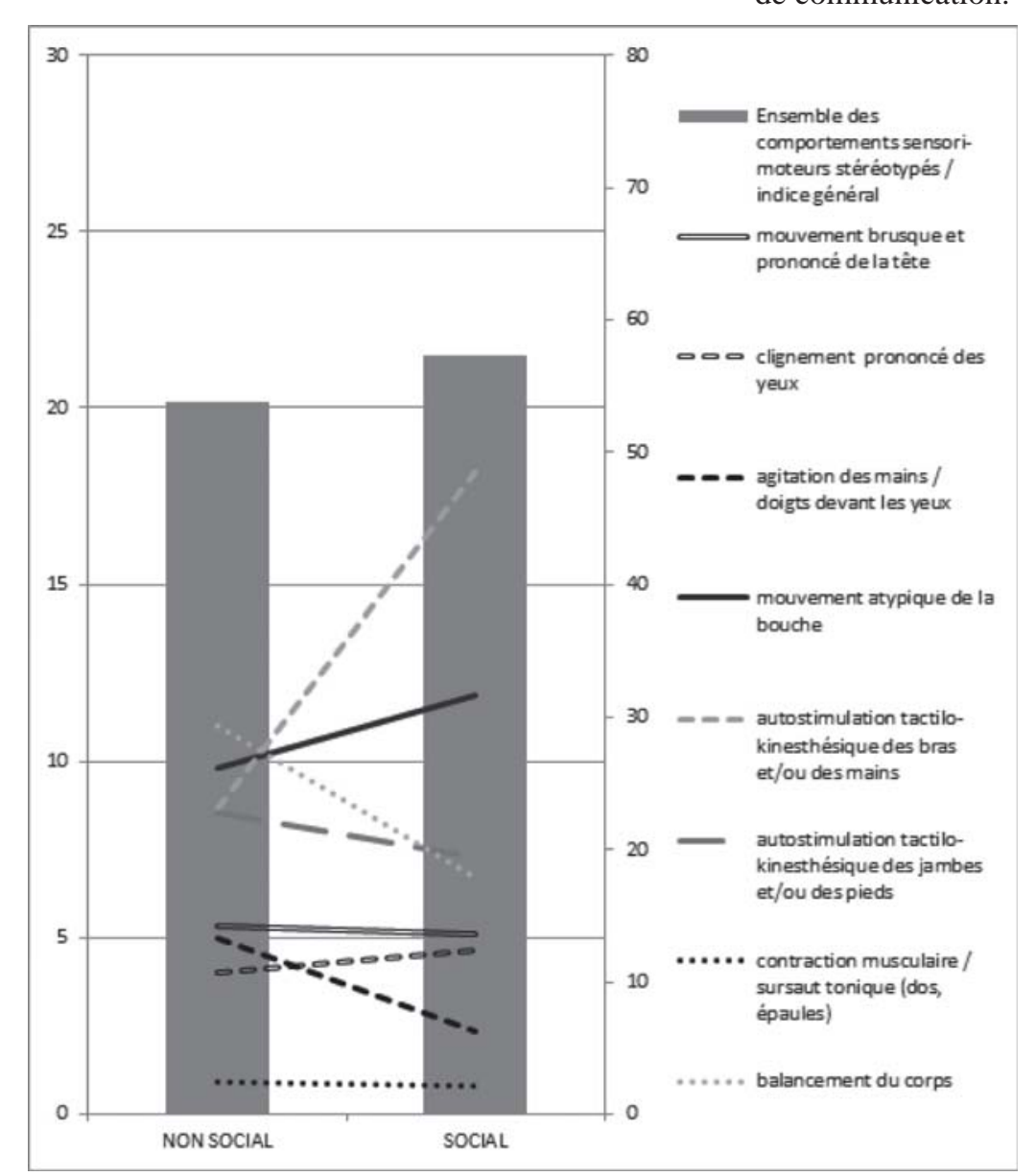

Graphique 7. Proportion temporelle de l'occurrence des principaux comportements sensorimoteurs stéréotypés relevés. Remarque : l'axe des ordonnées situé à droite du graphique correspond à la proportion temporelle de l'occurrence

de l'ensemble des comportements sensorimoteurs stéréotypés (indice général).
Nous remarquons, tout d'abord, que la prévalence des manifestations émotionnelles positives de Sylvie est significativement plus importante lors de la présentation des stimuli de nature sociale (graphique 6 et tableau 8), alors que la prévalence de l'ensemble des comportements sensorimoteurs stéréotypés (indice général) ne varie que très peu selon la nature sociale ou non sociale des stimuli présentés (graphique 7 et tableau 9), tout comme les indices généraux de modification des différents flux sensoriels (graphique 8 et tableau 10). La différence la plus manifeste se rapporte au comportement d'autostimulation tactilo-kinesthésique des bras ou des mains, dont la prévalence double dans la modalité « social ». Ainsi, on remarque que ce comportement et celui de mouvement des lèvres sont ceux qui présentent les plus fortes prévalences lors de la présentation de stimuli de nature sociale : ces observations peuvent faire supposer que des stimulations de nature sociale peuvent entraîner, chez Sylvie, une activation accrue de ses organes de communication. 


\begin{tabular}{|c|c|c|c|c|c|}
\hline & \\
\hline & Tactile & Visuel & Auditif & $\begin{array}{l}\text { Vesti- } \\
\text { bulaire }\end{array}$ & $\begin{array}{l}\text { Visuel- } \\
\text { auditif }\end{array}$ \\
\hline $\begin{array}{l}\text { Ensembles des comportements sensorimo- } \\
\text { teurs stéréotypés/indice général }\end{array}$ & 56,63 & 58,74 & 59,74 & 72,33 & 75,62 \\
\hline Mouvement brusque et prononcé de la tête & 3,82 & 4,51 & 6,73 & 5,58 & 7,96 \\
\hline Clignement prononcé des yeux & 4,38 & 4,10 & 4,54 & 5,84 & 5,97 \\
\hline Agitation des mains / doigts devant les yeux & 1,57 & 2,46 & 0,15 & 4,82 & 2,24 \\
\hline Mouvement atypique de la bouche & 13,15 & 9,27 & 10,10 & 8,12 & 15,92 \\
\hline $\begin{array}{l}\text { Autostimulation tactilo-kinesthésique des } \\
\text { jambes ou des pieds }\end{array}$ & 18,09 & 7,79 & 3,95 & 10,91 & 11,69 \\
\hline $\begin{array}{l}\text { Contraction musculaire / sursaut tonique } \\
\text { (dos, épaules) }\end{array}$ & 0,45 & 0,98 & 0,58 & 0,76 & 0,75 \\
\hline Balancement du corps & 1,80 & 12,55 & 18,59 & 0 & 17,91 \\
\hline
\end{tabular}

Tableau 9. Proportion temporelle de l'occurrence des principaux comportements sensorimoteurs stéréotypés relevés.

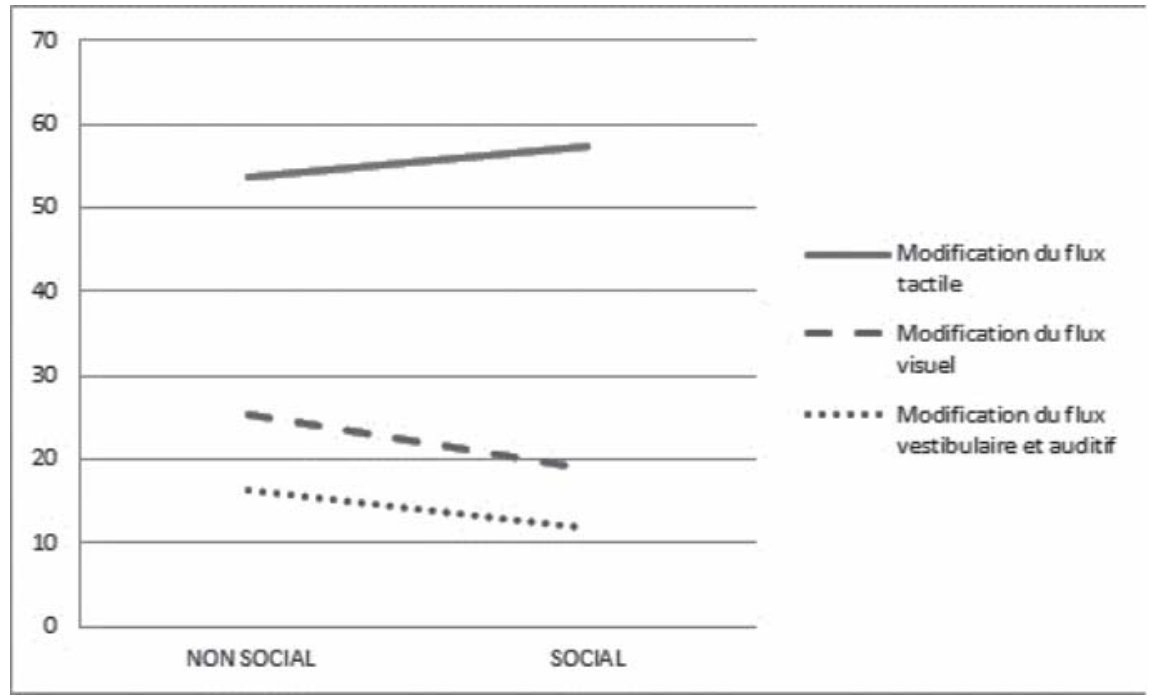

Graphique 8. Indice de modification des flux sensoriels par les stéréotypies sensorimotrices du sujet.

\begin{tabular}{lcc}
\cline { 2 - 3 } & Non social & Social \\
\hline Modification du flux tactile & 53,75 & 57,30 \\
\hline Modification du flux visuel & 25,33 & 18,77 \\
\hline $\begin{array}{l}\text { Modification du flux vestibulaire } \\
\text { et auditif }\end{array}$ & 16,35 & 11,80 \\
\hline
\end{tabular}

Tableau 10. Indice de modification des flux sensoriels par les stéréotypies sensorimotrices du sujet.

\section{SYNTHÈSE DES RÉSULTATS \\ DE L'ENSEMBLE DE LA RECHERCHE}

Nous présentons, à présent, une synthèse des principaux résultats, observations et analyses, issus de la mise en application de notre méthodologie de recherche qui nous a conduits à établir le profil corporel (une schématisation de la corporéité) de cinq sujets (dont celui de Sylvie détaillé précédemment et synthétisé par la figure 1), ceci nous permettant une objectivation et formalisation de nos observations quant à leur vécu et fonctionnement corporels en situation.

Pour l'ensemble des sujets, nous avons relevé un investissement prioritaire des modalités sensorielles proximales (sens tactile, kinesthésique et vestibulaire) par rapport aux modalités sensorielles 


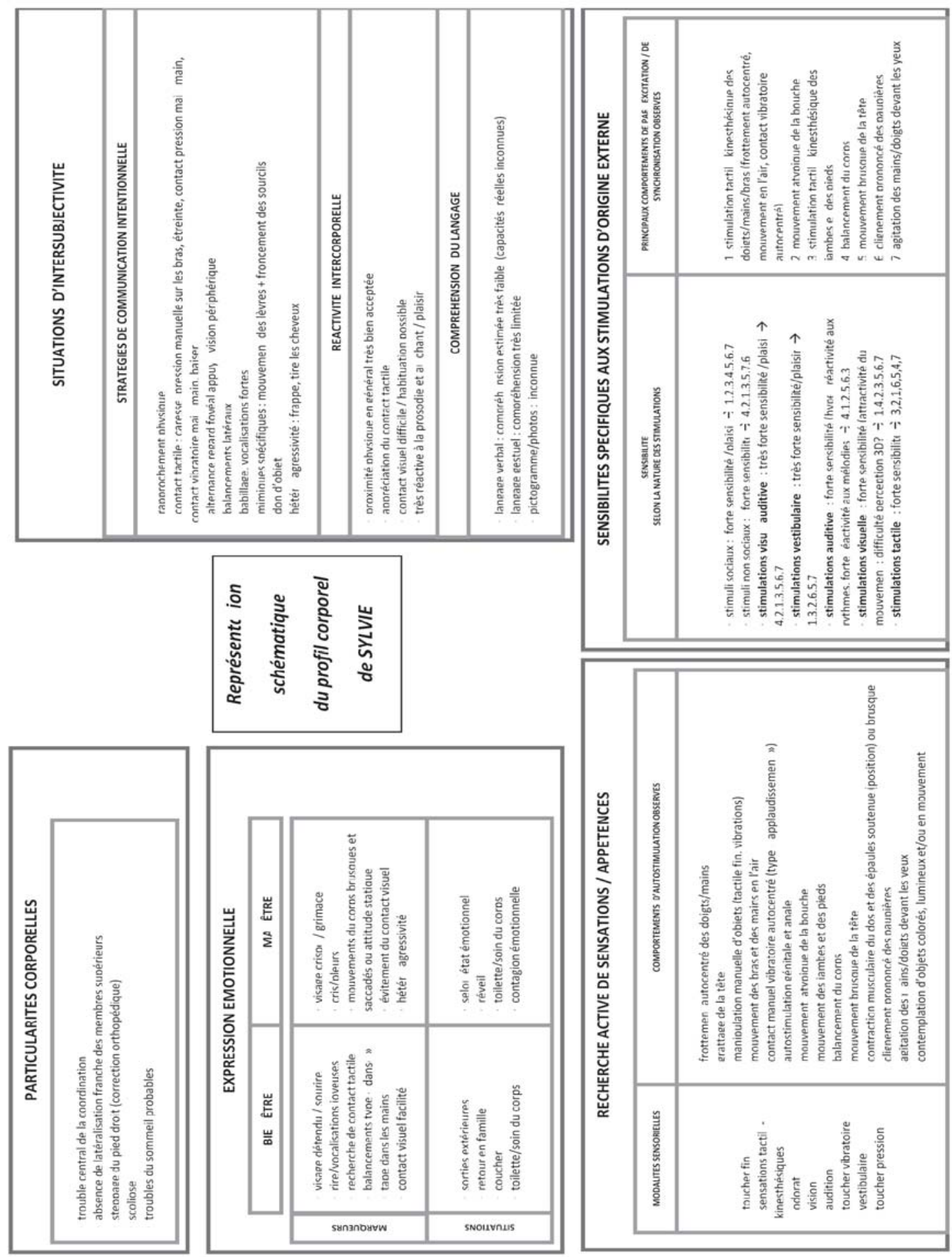

Figure 1. Présentation schématique du profil corporel de Sylvie (les chiffres indiqués dans la catégorie «Sensibilité selon la nature des stimulations » indiquent les comportements sensorimoteurs stéréotypés observés dans les situations indiquées, présentés selon leurs prévalences respectives, dans un ordre décroissant). 
distales (principalement la vision et l'audition), en accord avec la littérature (Bellusso, Desnos, Segond, 2014 ; Bellusso, Maumy-Bertrand, Desnos, Segond, 2014 ; Bullinger, 2008 ; O’Rirodan, Passetti, 2006). Ceci nous a semblé manifeste, tant au niveau des multiples stéréotypies sensorimotrices observées, impliquant majoritairement la sensibilité tactilo-kinesthésique (mouvement des mains et des doigts, par exemple), qu'à celui des modalités de communication intentionnelle observées envers autrui (principalement effectuée par rapprochement et contact tactile).

En second lieu, nous avons pu observer que tous les sujets semblaient présenter une forte sensibilité au contexte sensoriel et social, manifeste principalement à travers les modifications de la fréquence ou de l'intensité des stéréotypies sensorimotrices. Celles-ci augmentent tout particulièrement face à des stimulations distales environnementales (stimulations visuelles et auditives, de nature sociale comme non sociale), ainsi que lors de stimulations sociales (regard dirigé, parole adressée, rapprochement physique).

Par conséquent, les sujets présentent une grande sensibilité et vulnérabilité à l'attitude des accompagnants (attention dirigée et intérêt manifeste, modalités de communication utilisées...), qui se manifestent notamment par l'expression de stéréotypies sensorimotrices (plus ou moins apparentes).

\section{CONCLUSION ET DISCUSSION}

Nous avons choisi de centrer notre démarche sur la corporéité des personnes autistes institutionnalisées en FAM, avec retard mental profond associé, en majorité non verbales, comme base de compréhension de leur subjectivité, difficilement accessible, mais dont le respect est néanmoins primordial dans le cadre de tout accompagnement psychologique. Cet accompagnement, conformément au code de déontologie de la profession, doit, en premier lieu, être respectueux des différences, attentes, besoins, choix et refus de ces personnes, en vue de favoriser leur bien-être au quotidien, dans une perspective de bientraitance.

L'ensemble de nos observations nous amène à conclure que la bientraitance des personnes AA-DIP, accompagnées en structure médico-sociale de type FAM, nécessite impérativement une connaissance adaptée des particularités de leur vécu et fonctionnement corporels, dont la nature singulière et qualitativement différente doit être étudiée précisément. L'hétérogénéité des problématiques de ces sujets nous parait impliquer nécessairement une étude individualisée et contextualisée de leur cognition incarnée, qui devrait être systématiquement conduite et soutenue par le personnel cadre de l'institution.

Or, nous avons relevé une difficulté majeure, chez la plupart des accompagnants, à pouvoir avoir une compréhension non «normo-centrée » de la corporéité des sujets. Il apparaît, en effet, très difficile, pour ces professionnels, de soutenir ce que nous nommerons une position « méta » en situation d'interaction avec les personnes accompagnées, c'està-dire de considérer que le vécu et le fonctionnement corporels des sujets peut être qualitativement différent des leurs et d'agir en conséquence. Cette difficulté paraît résulter, chez les accompagnants, d'une automatisation du processus d'empathie, ainsi que de leur méconnaissance de la corporéité de chaque sujet.

Aussi, le personnel accompagnant, dont l'attitude et le comportement nous semblent constituer le facteur premier et déterminant pouvant contribuer à la bientraitance des sujets, doit-il être impérativement formé à soutenir une approche non normo-centrée de la corporéité des sujets, ce que nous semblent pouvoir permettre, notamment, les formations de type médiation corporelle et Snoezelen. L'objectif étant d'accéder à leur subjectivité et à l'objectiver en maîtrisant la nôtre afin de pouvoir réellement comprendre et respecter leurs différences et leurs choix. Dans cette perspective, les modalités d'une intervention brève systémique auprès d'une équipe d'accompagnants, centrée sur la problématique d'un sujet spécifique, seront traitées dans d'autres articles ultérieurement.

Outre la prise en compte de la subjectivité des personnes AA-DIP, via leur corporéité et de la dynamique relationnelle et intersubjective accompagnant-sujet, la grande vulnérabilité de ces personnes, sur le plan de leur intégrité corporelle et psychique et leur extrême sensibilité à leur environnement sensoriel et social, nous invitent également à considérer, dans l'optique de bientraitance de ces personnes, deux autres niveaux d'analyse. D'une part, l'identification des problèmes liés à la situation de groupe (le niveau groupal, le vécu des sujets étant, comme nous l'avons indiqué, quasi constamment tributaire des situations groupales, avec une composition des groupes qui varie fréquemment au cours de la journée) et, d'autre part, l'identification de problèmes liés à l'organisation du FAM (le niveau institutionnel). Ces deux niveaux d'analyse seront, quant à eux, également traités ultérieurement. 
Enfin, du fait de la complexité de la situation d'accompagnement dans de telles structures, il nous paraît également indispensable que le personnel soit soutenu régulièrement sur le plan psychologique.

\section{Remerciements}

Les auteurs remercient le Foyer d'accueil médicalisé Pierre Valdo de Marmoutier et la Fondation Sonnenhof de Bischwiller pour leur collaboration, ainsi que le Conseil scientifique de l'université de Strasbourg et le Conseil régional d'Alsace pour le financement de cette recherche.

\section{RÉFÉRENCES}

Abric (Jean-Claude).- Exclusion sociale, insertion et prévention, Toulouse, Érès, 2003.

Agence nationale de l'évaluation et de la Qualité DES ÉTABLISSEMENTS ET SERVICES SOCIAUX ET MÉDICAUX SOCIAUX.- Recommandations de bonnes pratiques professionnelles. La bientraitance : définition et repères pour la mise en œuvre ANESM, 2008. Disponible sur http:// www.anesm.sante.gouv.fr/

Aitken (Kenneth J.), Trevarthen (Colwyn).- L'organisation soi-autrui dans le développement psychologique humain, Psychiatrie de l'enfant, 46, 2, 2003, p. 471-520.

Bellusso (Perrine), Desnos (Yves), Segond (Hervé).Intérêts de la psychothérapie à médiation sensorielle dans le cadre de la prise en charge des troubles de la relation et de la communication chez des enfants autistes sévèrement déficitaires I : Introduction aux applications cliniques, Neuropsychiatrie de l'enfance et de l'adolescence, 62, 2, 2014, p. 90-94.

Bellusso (Perrine), Maumy-Bertrand (Myriam), Desnos (Yves), Segond (Hervé).- Intérêts de la psychothérapie à médiation sensorielle dans le cadre de la prise en charge des troubles de la relation et de la communication chez des enfants autistes sévèrement déficitaires II : Illustration clinique, Neuropsychiatrie de l'enfance et de l'adolescence, 62, 2, 2014, p. 95-101.

Blondel (Frédéric).- Un mode spécifique d'accompagnement des personnes polyhandicapées enfants et adultes en institution : la pratique Snoezelen, Paris, Centre de ressources multihandicap, 2003.

Bourreau (Yannig).- Les comportements répétés et restreints dans l'autisme : construction et validation d'une échelle d'évaluation, thèse de doctorat non publiée, Université François-Rabelais, Tours, 2008.

Bovet (Pierre).- Identité crevassée et identité menacée, Psychothérapies, 25, 4, 2005, p. 247-252.

BULLinger (André).- Le développement sensori-moteur de l'enfant et ses avatars. Un parcours de recherche, Toulouse, Érès, 2008.

Calpini (Jean-Claude), Cardinet (Jean), Dominicé (Pierre), Muller (Charles), Patry (Jean-Luc), Perret (Jean-François), PINI (Gianreto), WeISS (Jacques).Recherche-action : interrogations et stratégies émergentes, Cahiers de la section des sciences de l'éducation, Pratiques et théorie, 26, 1981 [Le 19 octobre 2018, http:// archive-ouverte.unige.ch/unige:33397].
Cascio (Carissa J.).- Somatosensory processing in neurodevelopmental disorders, Journal of Neurodevelopmental Disorders, 2, 2010, p. 62-69.

Chamak (Brigitte).- Modèles de la pensée : quels enjeux pour les chercheurs en sciences cognitives ?, Intellectica, 2, 39, 2004, p. 79-105.

Chamak (Brigitte), Bonniau (Béatrice), Jaunay (Emmanuel), CoHEN (David).- What can we learn about autism from autistic persons?, Psychotherapy and Psychosomatics, 77, 5, 2008, p. 271-279.

Снамак (Brigitte), Cohen (David).- L'autisme : vers une nécessaire révolution culturelle, Médecine/Sciences, 19, 11, 2003, p. 1152-1159.

Chavaroche (Philippe).- Les personnes en situation de grande dépendance : une question de limites ? Reliance, 21, 3, 2006, p. 35-41.

CIM-11 - Classification statistique internationale des maladies et des problèmes de santé connexes, $11^{e}$ révision, Organisation mondiale de la santé, 2018.

COSNIER (Jacques).- Observation directe des interactions précoces, ou les bases de l'épigénèse interactionnelle, Psychiatrie de l'enfant, 27, 1, 1981, p. 107-126.

Cosnier (Jacques), Chavarel (Marie).- Développement théorico-clinique du concept d'épigénèse interactionnelle, Psychiatrie française, XXIX, 3, 1998, p. 79-87.

Courtors (Alain).- Les fondamentaux de la recherche systémique en psychologie clinique, Thérapie familiale, 30, 2, 2009, p. 275-288.

DiRECTION DE LA RECHERCHE, DES ÉTUdes, DE L'ÉVALUATION ET DES STATISTIQUES (DREES).- Les foyers d'accueil médicalisé. Établissements et services pour adultes handicapés - Résultats de l'enquête ES 2006 et séries chronologiques 1995 à 2006. Série statistiques $\mathrm{N}^{\circ} 141$, 2010. Consultable sur : http://www.sante.gouv.fr/IMG/ pdf/ seriestat141-t2-5.pdf

DSM-5.- Diagnosis and Statistical Manual of Mental Disorders, Washington DC, American Psychiatric Association, $5^{\text {e }}$ édition révisée, 2013.

Fombonne (Éric), Achard (Sophie), Tuffreau (Renée).L'évaluation du comportement adaptatif : l'échelle de Vineland, Handicap et inadaptations, les Cahiers $d u$ CTNERHI, 1995, p. 67-68, 79-89 (version originale publiée en 1984 sous le titre Vineland Adaptative Behavior Scales (VABS), Sparrow, Balla, Cicchetti, Circle Pines: American Guidance Service). 
Frith (Uta)-- L'énigme de l'autisme, Paris, Odile Jacob, 2010

Fuchs (Thomas), De JAEGHer (Hanne).- Enactive intersubjectivity: Participatory sense-making and mutual incorporation, Phenomenoly and the Cognitive Sciences, 8, 2009, p. 465-486.

Garbarani (Francesca), Adenzato (Mauro).- At the root of embodied cognition: Cognitive science meets neurophysiology, Brain and Cognition, 56, 2004, p. $100-106$

GEPNER (Bruno).- Constellation autistique, mouvement, temps et pensée. Malvoyance de l'É-Motion, autres désordres du traitement temporospatial des flux sensoriels et dyssynchronie dans l'autisme, Devenir, 18, 4, 2006a, p. 333-379.

GePNER (Bruno).- Le monde va trop vite pour les personnes autistes ! Hypothèses neurophysiopsychopathogéniques et implications rééducatives, Neuropsychiatrie de l'enfance et de l'adolescence, 54, 2006b, p. 371-374

GEPNER (Bruno).- Réseau adultes autistes-famillesprofessionnels-institutions : quelques erreurs à éviter et propositions, Bulletin scientifique de l'Arapi, 30, 2012, p. 25-27.

GEPNER (Bruno).- Autismes. Ralentir le monde extérieur, calmer le monde intérieur, Paris, Odile Jacob, 2014.

Grèzes (Julie), De Gelder (Béatrice).- Contagion motrice et émotionnelle, dans Andres (C.), Barthélémy (C.), Berthoz (A.), Massion, (J.), Rogé (B.), Autisme, cerveau et développement : de la recherche à la pratique, Paris, Odile Jacob, 2005, p. 295-319.

HAMONET (Claude).- Les personnes en situation de handicap, Paris, Presses universitaires de France, $6^{e}$ éd. 2010.

HAPPÉ (Francesca), Frith (Uta).- The Weak Coherence Account: Detail-focused Cognitive Style in Autism Spectrum Disorders, Journal of Autism and Developmental Disorders, 36, 1, 2006, p. 5-25.

Haute autorité DE SAnté.- Trouble du spectre de l'autisme : interventions et parcours de vie de l'adulte. Recommandations de bonnes pratiques professionnelles, 2017. [Le 19 octobre 2018, https://www.has-sante.fr/ portail/jcms/c_1101438/fr/tableau-des-recommandationsou-travaux-relatifs-a-la-bonne-pratique]

Hochmann (Jacques).- Pour soigner l'enfant autiste, Paris, Odile Jacob, 1997.

Hulsegge (Jan), Verheul (Ad).- Snoezelen: Another World, Londres, Rompa, 1988.

Hutchins (Edwin).- Enaction, Imagination, and Insight, dans Stewart (J.), Gapenne (O.), Di Paolo (E.A.), Enaction. Toward a New Paradigm for Cognitive Science, Cambridge, Londres, A Bradford Book, The MIT Press, 2010, p. 425-250.

IAROCCI (Grace), McDonald (John).- Sensory integration and the perceptual experience of persons with autism, Journal of Autism and Developmental Disorders, 36, 1, 2006, p. 77-90.

KANNER (Leo).- Autistic Disturbances of Affective Contact, Nervous Child, 2, 1943, p. 217-250.

Kaufmann (Laurence), Clément (Fabrice).- Is socio- logy core knowledge? The social nature of the cognitive architecture, Intellectica, 36, 2003, p. 421-457.

Kloeckner (Anja), Jutard (Claire), Bullinger (André), Nicoulaud (Lucie), Tordjman (Sylvie), Cohen (David).Intérêt de l'abord sensorimoteur dans les pathologies autistiques sévères I : introduction aux travaux d'André Bullinger, Neuropsychiatrie de l'enfance et de l'adolescence, 57, 2, 2009, p. 154-159.

Laplanche (Jean), Pontalis (Jean-Bertrand).- Vocabulaire de la psychanalyse, Paris, Presses universitaires de France, 1967.

Mottron (Laurent), Dawson (Michelle), Soulières (Isabelle), Hubert (Benedicte), Burack (Jack).--Enhanced Perceptual Functioning in Autism: An Update, and Eight Principles of Autistic Perception, Journal of Autism and Developmental Disorders, 36, 1, 2006, p. 27-43.

NADEL (Jacqueline).- Le futur des émotions : un nécessaire tressage des données normatives et psychopathologiques, Enfance, 55, 2003, p. 23-32.

NADEl (Jacqueline), Potier (Caroline).- Imitez, imitez, il en restera toujours quelque chose : le statut développemental de l'imitation dans le cas d'autisme, Enfance, 54, 1, 2002, p. 76-85.

Niedenthal (Paula M.).-Embodying Emotion, Science, 316, 2007, p. 1002-1005.

O'Rirodan (Michelle), Passetti (Filippo).- Discrimination in autism within different sensory modalities, Journal of autism and developmental desorders, 36, 2006, p. 665-675.

Pelloux (Anne-Sylvie), Delion (Pierre), Tessarech (Sarah).- Introduction, Enfances et Psy, 46, 1, 2010, p. 10-14.

Penelaud (Olivier).- Le paradigme de l'énaction aujourd'hui. Apport et limites d'une théorie cognitive « révolutionnaire », Plastir, 18, 1, 2010, p. 1-35.

Pernon (Éric), Pry (René), Baghdadli (Amaria).Autism: Tactile perception and emotion, Journal of Intellectual Disability Research, 51, 8, 2007, p. 580-587.

Pernon (Éric), Rattaz (Cécile).- Les modes d'expression de la douleur chez l'enfant autiste : étude comparée, Devenir, 15, 3, 2003, p. 263-277.

ReCORDON-GABORIAUd (Séverine).- L'évaluation clinique des adultes avec autisme et déficience intellectuelle associée : une nécessité en faveur d'un accompagnement personnalisé, Annales médico-psychologiques, 170, 2012, p. 485-490.

Rizzolatti (Giacomo), Fabbri-Destro (Maddalena), CAttaneo (Luigi).- Mirror neurons and their clinical relevance, Nature Clinical Practice: Neurology, 5, 1, 2009 , p. 24-34.

Rizzolati (Giacomo), Sinigaglia (Corrado).- Les neurones miroirs, Paris, Odile Jacob, 2008.

SCHOpler (Eric), Reichler (Robert Jay), RochenRENNER (Barbara).- Échelle d'évaluation de l'autisme infantile. (B. Rogé traduction et adaptation française), Issy-les-Moulineaux, EAP, 1989, p. 213-224 (version originale publiée en 1980 sous le titre Toward objective classification of childhood autism: Childhood Autism Rating Scale (CARS), Schopler, Reichler, De Vellis, Daly, Journal of Autism and Developmental Disorders, 10, p. 91-103). 
Sebbah (François-David).- L'usage de la méthode phénoménologique dans le paradigme de l'enaction, Intellectica, 39, 2004, p. 169-188.

SEGOND (Hervé).- Le toucher en développement : latéralité, cécité, autisme. Des activités perceptives au soin, Sarrebruck, Éditions universitaires européennes, 2015.

Segond (Hervé), Maris (Stéphane), Desnos (Yves), Bellusso (Perrine).- IHM de suppléance sensorielle visuo-tactile pour aveugles et d'intégration sensorielle pour autistes, Journal d'interaction personne-système, 2 , 1, 2011, p. 2.1-2.15.

Segond (Hervé), Weiss (Déborah), Sampaio (Eliana).Human navigation via a visuo-tactile sensory substitution system, Perception, 34, 2005, p. 1231-1249.

Segond (Hervé), Weiss (Déborah), Sampaio (Eliana).A proposed Tactile-Stimulation System for Infants Who are Blind Tested on Sighted Infants, Journal of Visual Impairment and Blindness, 101, 1, 2007, p. 32- 43.

Segond (Hervé), Weiss (Déborah), Sampaio (Eliana).Perceiving space and optical cues via a visuo-tactile sensory substitution system: A methodological approach for training of blind subjects for navigation, Perception, 42, 2013, p. 508-528.

Sмiтh (Justin D.).- Single-Case Experimental Designs: A Systematic Review of Published Research and Current Standards, Psychological Methods, 2012, Advance online publication, doi: 10.1037/a0029312.

TARDIF (Carole).- Les particularités sensorielles des personnes autistes et leur incidence sur la vie quotidienne : des connaissances aux interventions, dans Tardif (C.), Autisme et pratiques d'intervention, Marseille, Solal Éditeur, 2010, p. 127-183

TARdif (Carole), Gepner (Bruno).- L'autisme, Paris, Dunod, $4^{\mathrm{e}}$ édition, 2014.

TARDIF (Carole), LATZKo (Laura), ARCisZEWSKi (Thomas), GEPNER (Bruno).- Reducing Information's Speed Improves Verbal Cognition and Behavior in Autism: A 2-Cases Report, Pediatrics, 139, 6, 2017, p. e1-e6.
Thомmen (Évelyne).- Les émotions chez l'enfant. Le développement typique et atypique, Paris, Belin, 2010.

Thommen (Évelyne), Chastellain (Amélie).- Les comportements stéréotypés chez trois personnes adultes atteintes d'autisme : une analyse de leur occurrence lors d'activité quotidienne, Revue francophone de la déficience intellectuelle, 20, 2009, p. 156-164.

Trevarthen (Colwyn).- Autism, sympathy of motives and music therapy, Enfance, 54, 1, 2003, p. 86-99.

Trevarthen (Colwyn), Aitken (Kenneth J.).- Intersubjectivité chez le nourrisson : recherche, théorie et application clinique, Devenir, 15, 4, 2003, p. 309-428.

Vaginay (Denis).- Découvrir les déficiences intellectuelles, Toulouse, Érès, 2007.

VARela (Francisco), Thompson (Evan), Rosch (Eléanor).-L'inscription corporelle de l'esprit. Sciences cognitives et expérience humaine, Paris, Éditions du Seuil, 1993.

Vygotski (Lev S.).- Pensée et langage, Paris, Éditions Sociales, 1985, réédition : Paris, La Dispute, 1997.

WASS (Sam).- Distortions and disconnections: Disrupted brain connectivity in autism, Brain and cognition, 75, 2011, p. 18-28.

Winkielman (Piotr), McIntosh (Daniel N.), Oberman (Lindsay).-Embodied and Disembodied Emotion Processing: Learning From and About Typical and Autistic Individuals, Emotion Review, 1, 2, 2009, p. 178-190.

\section{TEXTES DE LOI CITÉS}

Loi $\mathrm{n}^{\circ} 2002-2$ du 2 janvier 2002 rénovant l'action sociale et médico-sociale. Consultable sur www.legifrance.gouv.fr

Loi n 2005-102 du 11 février 2005, pour l'égalité des droits et des chances, la participation et la citoyenneté des personnes handicapées. Consultable sur www.legifrance. gouv.fr 